\title{
Modulation of fatty acid elongation in cockroaches sustains sexually dimorphic hydrocarbons and female attractiveness
}

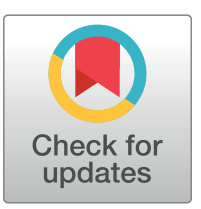

\section{Open ACCess}

Citation: Pei X-J, Fan Y-L, Bai Y, Bai T-T, Schal C, Zhang Z-F, et al. (2021) Modulation of fatty acid elongation in cockroaches sustains sexually dimorphic hydrocarbons and female attractiveness. PLoS Biol 19(7): e3001330. https://doi.org/ 10.1371/journal.pbio.3001330

Academic Editor: Richard Benton, University of Lausanne, SWITZERLAND

Received: March 26, 2021

Accepted: June 18, 2021

Published: July 27, 2021

Peer Review History: PLOS recognizes the benefits of transparency in the peer review process; therefore, we enable the publication of all of the content of peer review and author responses alongside final, published articles. The editorial history of this article is available here: https://doi.org/10.1371/journal.pbio.3001330

Copyright: @ 2021 Pei et al. This is an open access article distributed under the terms of the Creative Commons Attribution License, which permits unrestricted use, distribution, and reproduction in any medium, provided the original author and source are credited.

Data Availability Statement: PacBio data sets have been deposited in NCBl's SRA under the accession numbers SRR9143014 and SRR9143013. All BgElo sequences have been

\author{
Xiao-Jin Pei ${ }^{1}$, Yong-Liang Fan ${ }^{1 *}$, Yu Bai ${ }^{2}$, Tian-Tian Bai ${ }^{1}$, Coby Schal $\varpi^{3}$, Zhan- \\ Feng Zhang ${ }^{1}$, Nan Chen ${ }^{2}$, Sheng Li $\oplus^{2}$, Tong-Xian Liu $\oplus^{1}{ }^{*}$
}

1 State Key Laboratory of Crop Stress Biology for Arid Areas and Key Laboratory of Integrated Pest Management on Crops in Northwestern Loess Plateau, Ministry of Agriculture, Northwest A\&F University, Yangling, Shaanxi, China, 2 Guangdong Provincial Key Laboratory of Insect Developmental Biology and Applied Technology and Institute of Insect Science and Technology, School of Life Sciences, South China Normal University, Guangzhou, China, 3 Department of Entomology and Plant Pathology and W.M. Keck Center for Behavioral Biology, North Carolina State University, Raleigh, North Carolina, United States of America

* yfan@nwafu.edu.cn (Y-LF); txliu@nwafu.edu.cn (T-XL)

\section{Abstract}

Insect cuticular hydrocarbons (CHCs) serve as important intersexual signaling chemicals and generally show variation between the sexes, but little is known about the generation of sexually dimorphic hydrocarbons (SDHCs) in insects. In this study, we report the molecular mechanism and biological significance that underlie the generation of SDHC in the German cockroach Blattella germanica. Sexually mature females possess more $\mathrm{C} 29 \mathrm{CHCs}$, especially the contact sex pheromone precursor 3,11-DimeC29. RNA interference (RNAi) screen against the fatty acid elongase family members combined with heterologous expression of the genes in yeast revealed that both BgElo12 and BgElo24 were involved in hydrocarbon $(\mathrm{HC})$ production, but $B g E l o 24$ is of wide catalytic activities and is able to provide substrates for BgElo12, and only the female-enriched BgElo12 is responsible for sustaining femalespecific $\mathrm{HC}$ profile. Repressing BgElo12 masculinized the female $\mathrm{CHC}$ profile, decreased contact sex pheromone level, and consequently reduced the sexual attractiveness of female cockroaches. Moreover, the asymmetric expression of BgElo12 between the sexes is modulated by sex differentiation cascade. Specifically, male-specific $B g D s x$ represses the transcription of BgElo12 in males, while BgTra is able to remove this effect in females. Our study reveals a novel molecular mechanism responsible for the formation of SDHCs and also provide evidences on shaping of the SDHCs by sexual selection, as females use them to generate high levels of contact sex pheromone.

\section{Introduction}

Sexual dimorphism is prevalent in the animal kingdom. Females and males independently evolve some traits that enhance survival and reproduction under the pressure of divergent selection forces, thus leading to sexual dimorphism of traits such as body size [1]. Under sexual 
submitted to NCBI's GenBank under the accession numbers MT925720 and MW380216-MW380238.

Funding: This work was funded by the National Natural Science Foundation of China (http://www. nsfc.gov.cn), Grant No. 31772533 to YLF; the United States National Science Foundation (https:// www.nsf.gov), IOS-1557864 to CS and the Northwest A\&F University Special Foundation (https://www.nwsuaf.edu.cn), 2009-01-001-TXL to TXL. The funders had no role in study design, data collection and analysis, decision to publish, or preparation of the manuscript.

Competing interests: The authors have declared that no competing interests exist.

\begin{abstract}
Abbreviations: $A D$, adult day; CDS, complete coding sequence; $\mathrm{CHC}$, cuticular hydrocarbon; CRE, cis-regulatory element; Desat, desaturase; DMEM, Dulbecco's Modified Eagle Medium; Dmrt, doublesex/mab-3 related; dsRNA, double-stranded RNA; Dsx, doublesx, ELO, elongase; FA, fatty acid; FAME, fatty acid methyl ester; FAR, fatty acyl-CoA reductase; FAS, fatty acid synthase; GC-MS, gas chromatography-mass spectrometry; $\mathrm{HC}$, hydrocarbon; JH, juvenile hormone; LCFA, longchain fatty acid; PCA, principal component analysis; qPCR, quantitative PCR; RACE, 5' Rapid Amplification of CDNA Ends; $\mathrm{RH}$, relative humidity; RNAi, RNA interference; RT-PCR, real-time PCR; RT-qPCR, real-time quantitative PCR; SC-uracil, $S$. cerevisiae minimal medium minus uracil; SDCHC, sexually dimorphic cuticular hydrocarbon; SDHC, sexually dimorphic hydrocarbon; VLCFA, very long-chain fatty acid; WR, wing raising.
\end{abstract}

selection, asymmetric selection on the sexes can also result in the evolution of sexually dimorphic traits; however, these traits are subject to both inter- and intrasexual selection [2,3]. Because males and females of the same species share the majority of their genomes, the genetic basis of sex-specific traits that evolve under sexual selection is poorly understood. It is widely assumed that sexually dimorphic regulation of gene expression facilitates sex-specific adaptations [4-7]. Recruitment of preexisting genes or pathways into sexually dimorphic regulatory contexts has been proposed as a remarkable mechanism enabling the divergence of gene expression between the sexes [8-10]. The sex differentiation pathway is a conserved switch or regulator governing a set of downstream genes that direct sexually dimorphic traits $[11,12]$. The signaling cascades that transform sex differences (sex-specific chromosomes) into alternative splicing of sex differentiation genes (e.g., Transformer and Doublesex in insects) and the patterns of splicing these genes into sex-based isoforms have been widely described in different insect orders [13-17]. However, sexually dimorphic traits are usually generated from tightly associated multiple biosynthetic steps that are executed by a series of genes, and the nodes at which sex-determining signals connect with the biosynthetic pathway are poorly understood. Moreover, how these genes are translated into sexually dimorphic traits under elaborate spatial and temporal patterns also needs to be elucidated.

The main function of insect cuticular hydrocarbons ( $\mathrm{CHCs}$ ) is to waterproof the cuticle to resist dehydration under dry conditions [18]. In many insects, CHCs have been co-opted to serve as chemical signals (pheromones) that mediate intraspecific communication [19,20]. Sexually dimorphic cuticular hydrocarbon (SDCHC) profiles are widespread in insects [2123], but the regulatory networks that underlie the formation of SDCHCs largely remain unknown. Considerable progress has been made toward understanding the genetic basis of hydrocarbon (HC) biosynthesis and $\mathrm{CHC}$ variation in insects. An acetyl-CoA carboxylase catalyzes the biosynthesis of malonyl-CoA, and a cytosolic fatty acid synthase (FAS) incorporates malonyl-CoA units onto the acetyl-CoA primer to form linear long-chain fatty acids (LCFAs). A microsomal FAS catalyzes the biosynthesis of methyl-branched LCFAs using methylmalonyl-CoAs [24-30]. The LCF acyl-CoA can be selectively desaturated by a specific fatty acid desaturase (Desat), leading to unsaturated fatty acids [31,32]. The LCF acyl-CoAs are elongated to form very long-chain fatty acids (VLCFAs) with specific chain lengths by a cyclic fatty acid elongation system, including a rate-limiting elongase (ELO) and 3 other enzymes, elongase firstly incorporate a malonyl-CoA unite into the LCF acyl-CoA to generate a ketoacyl $\mathrm{CoA}$, the ketoacyl CoA is then reduced by a 3-keto-acyl-CoA-reductase to hydroxyacyl-CoA, which is dehydrated by a 3-hydroxy-acyl-CoA dehydratase to an enoyl-CoA, and further reduced by a trans-enoyl-CoA-reductase to yield the elongated VLCF acyl-CoA $[29,33]$. The VLCF acyl-CoAs are finally reduced to long-chain alcohols by fatty acyl-CoA reductases (FARs) and converted to HCs by the P450 oxidative decarbonylase CYP4Gs [34-36]. It is now clear that the variation in CHCs is primarily reflected in the chain length, number, and positions of methyl groups and the degree of unsaturation, which are determined by ELOs, FASs, and Desats, respectively [37-39]. These enzymes have been studied in few insect species with regard to sexually dimorphic hydrocarbons (SDHCs).

In the fruit fly Drosophila melanogaster, several studies have elucidated the pheromonal sexual dimorphism of CHCs. Female flies produce C27 and C29 dienes (7,11-heptacosadiene and 7,11-nonacosadiene), both of which function as female-specific contact sex pheromone components, whereas male flies produce $\mathrm{C} 23$ and C25 monoenes (7-tricosene and 7-pentacosene) [40]. Ferveur and colleagues found that the targeted expression of a sex differentiation gene, Transformer, in male oenocytes feminized the male CHC profile and elicited homosexual courtship from other males [41]. These findings implicated sex differentiation genes in the production of SDHCs. About 10 years later, Chertemps and colleagues revealed that the 
female-specific desat $F$ was responsible for the generation of pheromonal dienes in female $D$. melanogaster [31], and they also found that the sexual dimorphism in $\mathrm{HC}$ chain length was modulated by the female-specific eloF [33]. The specific expression of desatF in D. melanogaster females was due to a special cis-regulatory element (CRE) located upstream of desatF in D. melanogaster; the CRE presented a doublesex protein (Doublesx, Dsx) binding site that could be recognized by the female-specific isoform of $D s x(D s x-F)$, and the binding of Dsx-F activated the transcription of desatF, whereas male-specific Dsx showed no regulatory activity, resulting in sexual dimorphism of desatF expression [42]. However, the regulation of other genes involved in the production of SDHCs remains unknown in D. melanogaster, and studies in other insects are even rarer.

The German cockroach Blattella germanica is a significant worldwide indoor pest [43]. Considerable work has been done on biochemical aspects of CHCs in B. germanica [22,4446]. CHCs in B. germanica function as waterproofing agents [47], and as importantly, specific $\mathrm{HC}$ components are also the biosynthetic precursors for the production of contact sex pheromone components. The 3,11-DimeC29 is the most abundant $\mathrm{CHC}$ in females. Its hydroxylation at the 2 position, catalyzed by an age- and sex-specific putative cytochrome $\mathrm{P} 450$ and further oxidation of the- $\mathrm{OH}$, generates the main female-specific contact sex pheromone component, 3,11-DimeC29-2-one [48]. The contact sex pheromone is an efficient courtship signal. When a sexually mature male's antennae detect the sex pheromone on the female body surface, the male will raise his wings to expose a specialized tergal gland. Nutrients in the tergal secretion engage females in feeding, and the female is placed in an appropriate position for copulation [49-51]. More recently, Wexler and colleagues decoded the alternative splicing patterns of sex differentiation genes in B. germanica. BgTra is only functional in females and can splice $B g D s x$ into 2 nonfunctional female-specific isoforms $\left(B g D s x^{F}\right)$, whereas males generate a complete functional male-type $B g D s x\left(B g D s x^{M}\right)$ [52]. These findings provide opportunities for exploring the mechanisms underlying the SDHCs in B. germanica.

In this study, we employed B. germanica as a model insect. We characterize the SDCHCs, describe their temporal development, identify BgElo genes, and provide evidence that BgElo12 is responsible for the formation of SDCHC profiles. In addition, we found that the femaleenriched HCs are important in the generation of the female-specific contact sex pheromone; RNA interference (RNAi) of BgElo12 in females decreased courtship responses of males. Moreover, we show that BgElo12 is under the regulation of the sex differentiation pathway; $B g D s x^{M}$ can specifically repress the transcription of BgElo12. These findings suggest that the generation of SDHCs is achieved by placing a fatty acid elongation gene (BgElo12) under the regulation of $B g D s x^{M}$ and linking the sex differentiation regulatory cascade with the HC biosynthesis pathway, resulting in the asymmetric gene expression and SDHCs in B. germanica.

\section{Results}

\section{SDCHCs in B. germanica}

The temporal development of SDCHCs is rarely reported. In order to understand the molecular mechanisms of SDHC generation, we first analyzed the CHC profiles during sexual maturation. The oocytes of female cockroaches mature after eclosion by taking up vitellogenin until ovulation [45], and females become sexually receptive and mate 4 to 5 days before ovulation [53]. In our study, female cockroaches oviposited late on day 7 or early day 8 ; therefore, adult days 1 to 6 (AD1-6) adult cockroaches were used for CHC analysis. Different CHC components were identified as previously described [54]. We found no qualitative differences between males and females, but quantitative differences in CHCs became more apparent with adult age (S1 Table). At an early adult stage (AD1 and AD2), females and males had similar 
$\mathrm{CHC}$ profiles. However, differences were apparent at AD3 and gradually increased until AD6 (Fig 1A). Along with sexual maturation in males, the proportions of $\mathrm{C} 27 \mathrm{CHCs}$ and 9-; 11-; 13-; and 15-MeC29 (male-enriched peak 17) obviously increased, while other C29 CHCs, particularly 3,7-; 3,9-; and 3,11-DimeC29 (female-enriched peak 24), significantly decreased. In females, however, the $\mathrm{CHC}$ profiles consistently displayed high proportions of $\mathrm{C} 29 \mathrm{CHCs}$ and especially 3,7-; 3,9-; and 3,11-DimeC29 (Fig 1B-1E). Principal component analysis (PCA) showed that the male and female $\mathrm{CHC}$ profiles were more similar at AD1, but diverged at AD6, and the divergence was mainly reflected in principal component 2 , which largely represents the chain length factor (Fig $1 \mathrm{~F}$ and $1 \mathrm{G}$ ). The sexual dimorphism of CHCs was generated at the adult stage (nymphal $\mathrm{CHC}$ chromatogram showed no qualitative differences between males and females; S1 Fig), and the sexes diverged with sexual maturation. Notably, the differences between male and female $\mathrm{CHC}$ profiles suggested that chain length is an important factor in sexual dimorphism of CHCs. The putative $\mathrm{HC}$ biosynthetic pathway was shown in Fig $1 \mathrm{H}$. Our previous work identified a FAS gene (BgFas1) and a P450 oxidative decarbonylase gene (CYP4G19) that are involved in HC biosynthesis, but both showed no function in the formation of sexual dimorphism of CHCs $[55,56]$. However, the first step in elongation of LCF acyl-CoA is the key step to determine the final chain lengths of HCs. Therefore, we next identify BgElo genes in B. germanica.

\section{BgElo12 and BgElo24 are involved in HC biosynthesis}

We found that the differences of $\mathrm{CHCs}$ between females and males are largely reflected in carbon chain length. Based on this assumption, we searched for potential BgElo genes in the $B$. germanica genomic data and our full-length transcriptomic data [55,57]. A total of 24 different $B g E l o$ candidate genes were identified, and all BgElos were cloned and re-sequenced. Sequence alignment revealed that different BgElo proteins showed high homology and contained the conserved HXXHH and YXYY motifs [58]. All BgElo proteins displayed an ELO domain and several transmembrane domains (S1 Appendix).

The fatty acid precursors used for HC production generally originate in the oenocytes, but, also, can be transported from the fat body to oenocytes [29]. Therefore, we first analyzed the transcript levels of all BgElos in the fat body and abdominal integument. Results showed that BgElo24 and BgElo12 were highly expressed in the integument, and BgElo1, 2, 3, 6, 7, 9, 10, 11, $14,17,20$, and 22 were also abundant in the integument, while other BgElos were nearly undetectable (Fig 2A). In the fat body, BgElo10 and BgElo22 were highly expressed, BgElo1, 2, 3, 11, 12 , and 24 were slightly expressed, and other BgElos were undetectable (Fig 2B).

Based on these results, knockdown of the genes that were expressed in the abdominal integument or fat body was performed. A first injection of double-stranded RNA (dsRNA) was performed in early fifth-instar nymphs (N5D1 or N5D2), and a boost injection was performed 1 week later, and then the treated cockroaches were collected at different adult stages and subjected to $\mathrm{CHC}$ analysis. Knockdown of different BgElo genes significantly decreased the mRNA level (S2 Fig). Gas chromatography-mass spectrometry (GC-MS) analysis of CHCs showed that C27 CHCs were affected by many genes-knockdown of BgElo1, 10, 12, 14, 20, and 24 caused a significant increase in C27 CHCs, and repression of BgElo12 showed the greatest increase of the content of $\mathrm{C} 27 \mathrm{CHCs}$ (Fig 2C). C28 CHCs were rarely affected, with only knockdown of BgElo24 causing a significant decrease in C28 CHCs (Fig 2D). C29 CHCs were the most abundant in B. germanica, and only knockdown of BgElo12 or BgElo24 significantly decreased their amount (Fig 2E). Knockdown of BgElo12 or BgElo24 also dramatically decreased the amount of C30 CHCs, while knockdown of BgElo2 increased C30 CHCs (Fig 2F). CHCs with chain lengths greater than 30 occur in low quantities in B. germanica, but the 
Male

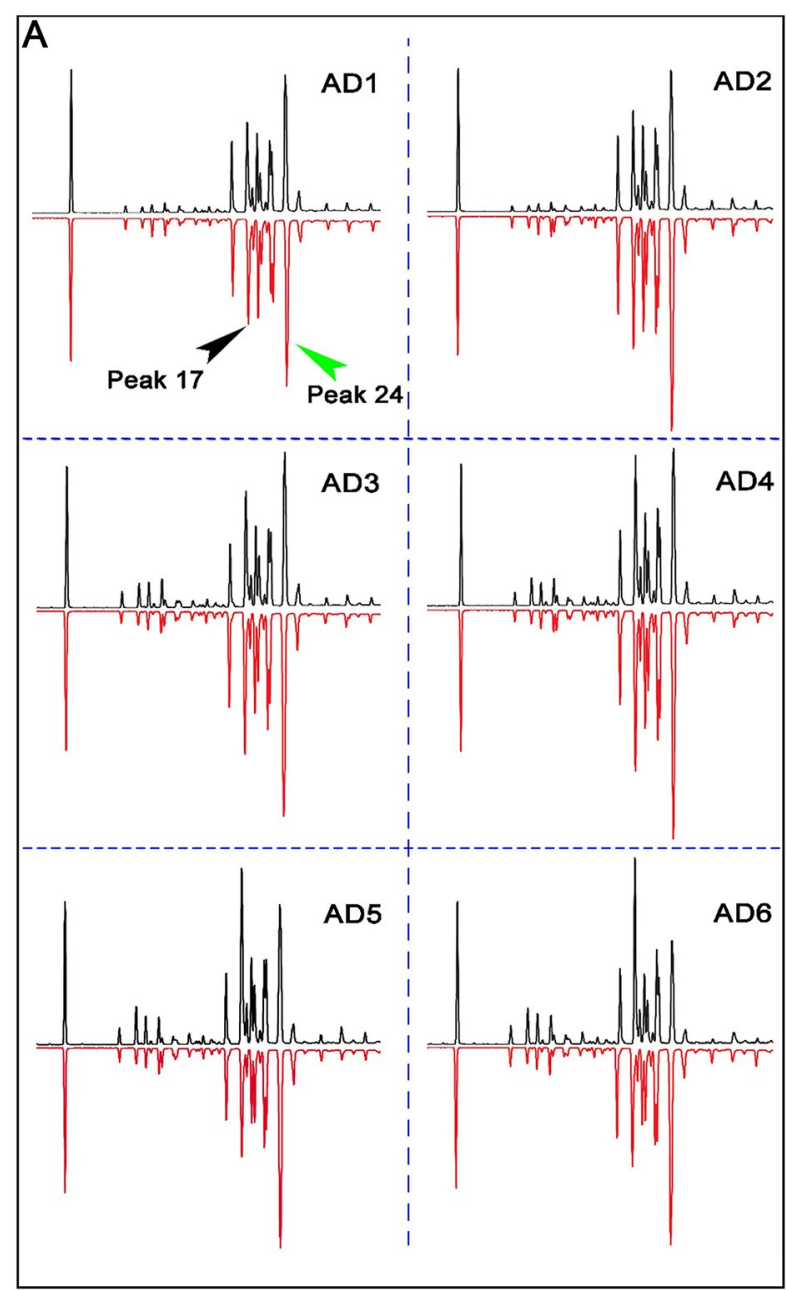

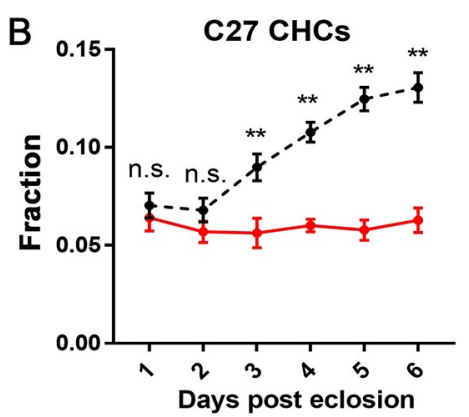

D

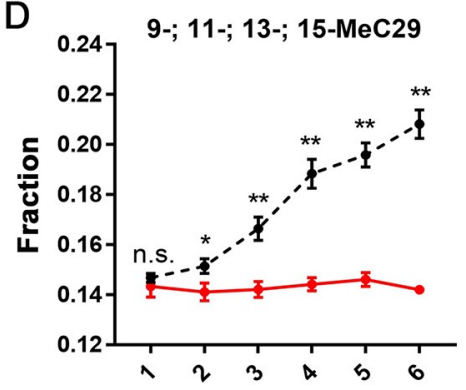

$\mathrm{F}$

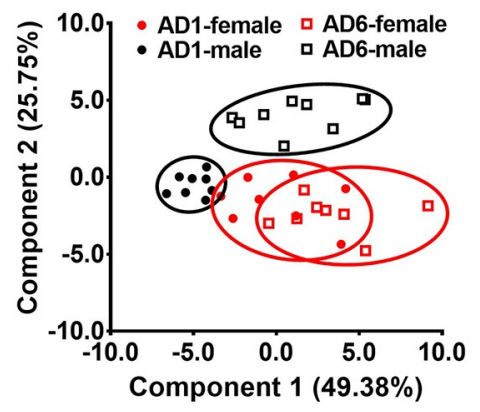

Female

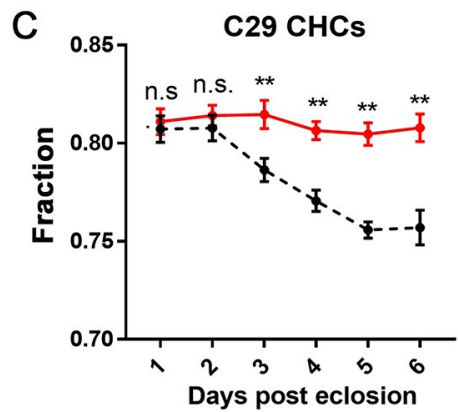

$\mathrm{E}$
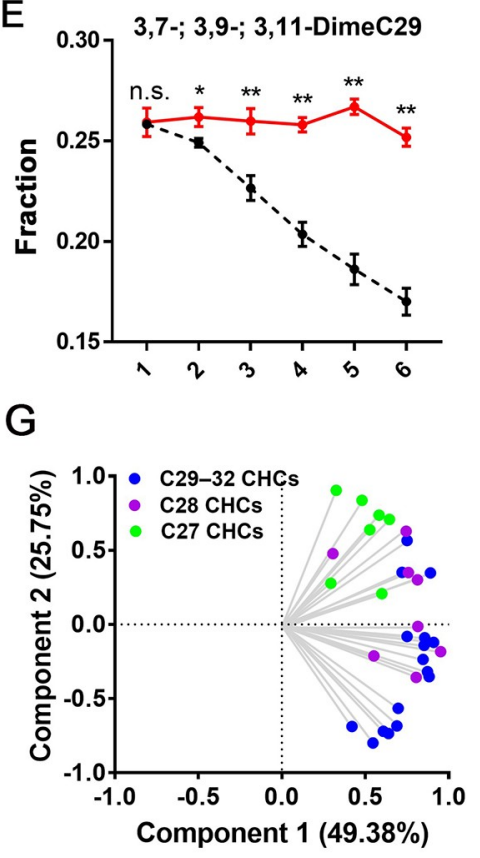

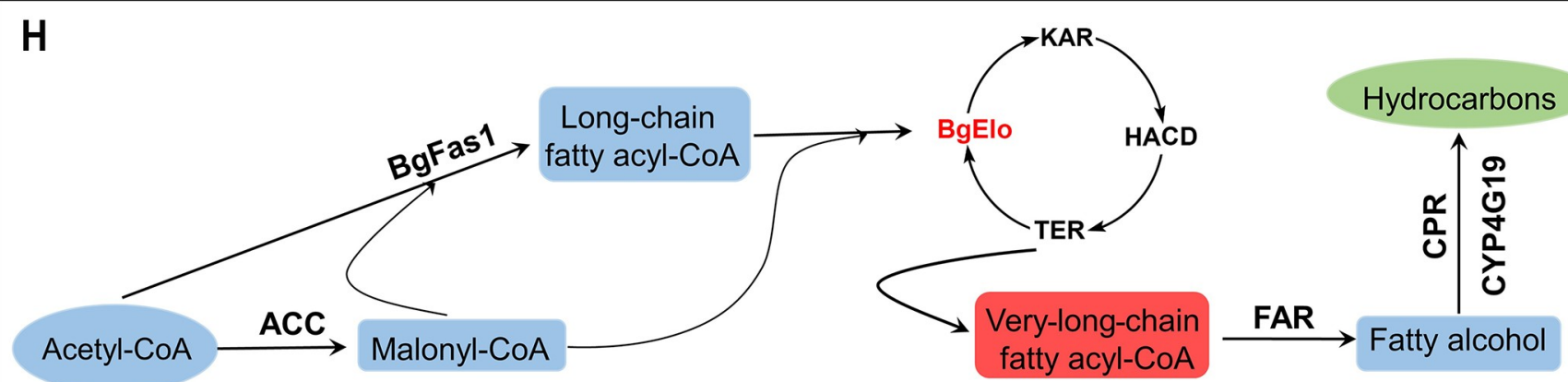

Fig 1. Development of sexually dimorphic CHCs. (A) Comparisons of AD1-AD6 male (black) and female (red) CHC profiles. The first peak represents the internal standard $n$-hexacosane. Other peaks correspond to the CHCs in S1 Table. Peak 17 is male enriched (9-; 11-; 13-; and 15-MeC29) and gradually increased with age in males. Peak 24 is female enriched (3,7-; 3,9-; and 3,11-DimeC29). The proportions of C27 CHCs (B), C29 CHCs (C), Peak 17 (D), and Peak 24 (E) in the total CHCs from AD1 to AD6 were compared between males (black dashed lines) and females (solid red lines). Data are shown as mean \pm SEM (n.s. represents no significant difference; ${ }^{*} P<0.05,{ }^{* *} P<0.01,2$-tailed Student $t$ test, $n=8-10$ ). (F, G) PCA of AD1 and AD6 CHC profiles of males and females. In (F), each dot represents a datum calculated from one cockroach. AD1 male (black solid circles) and female (red solid circles) $\mathrm{CHC}$ profiles overlapped, whereas AD6 male (black open square) and female (red open square) CHC profiles separated along PC2. In the loading diagram (G), CHCs with different chain lengths are marked by green (C27), purple (C28), and blue (C29-C32), showing that C27 CHCs vectored toward males along PC2, whereas longer chain CHCs vectored toward females. (H) Putative HC biosynthetic pathway in B. germanica. The data underlying this figure are included in S1 Table and S1 Data. ACC, acetyl-CoA carboxylase; AD, adult day; BgElo, fatty acid elongase from B. germanica; BgFas1, a FAS from B. germanica involved in 
HC biosynthesis; CHC, cuticular hydrocarbon; CPR, NADPH-cytochrome P450 reductase; CYP4G19, a P450 oxidative decarbonylase from B. germanica involved in HC biosynthesis; FAR, fatty acyl-CoA reductase; FAS, fatty acid synthase; HACD, 3-hydroxy-acyl-CoA dehydratase; HC, hydrocarbon; KAR, 3-keto-acyl-CoA reductase; PCA, principal component analysis; TER, trans-enoyl-CoA reductase.

https://doi.org/10.1371/journal.pbio.3001330.g001

A

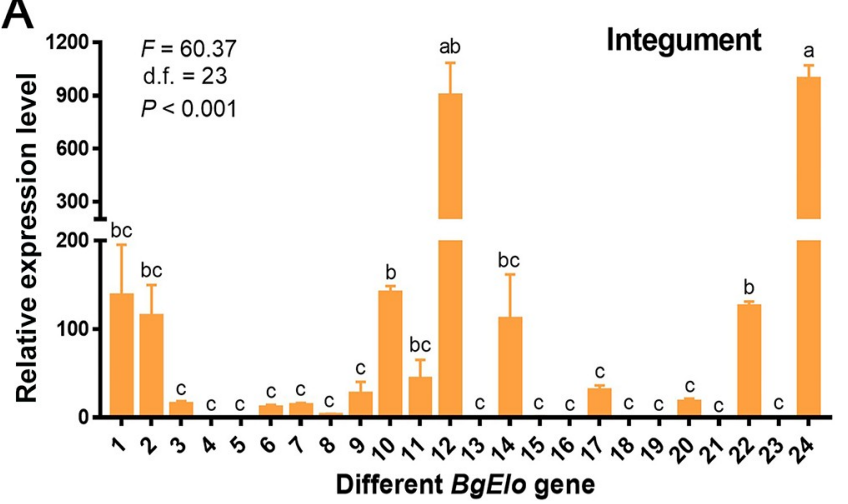

C

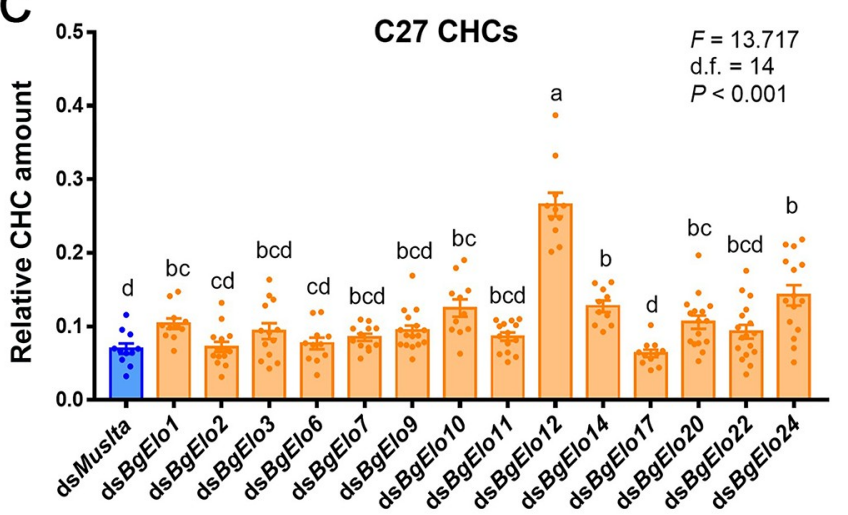

E

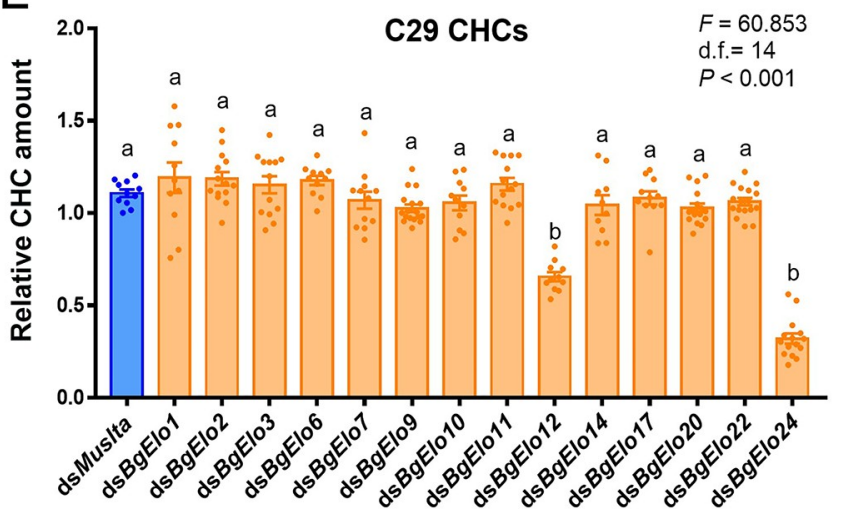

B

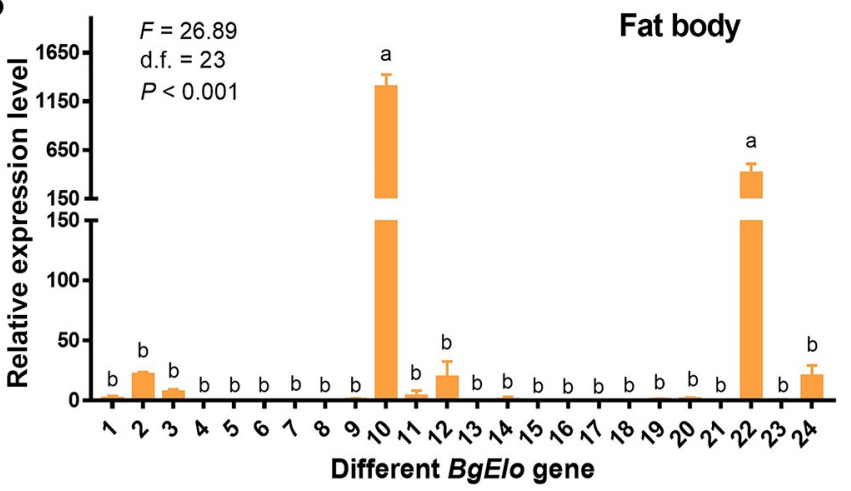

D

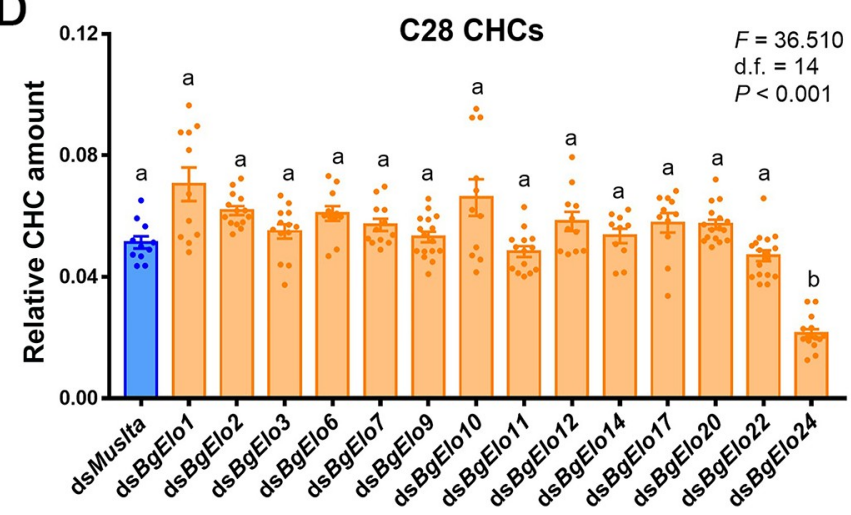

$\mathrm{F}$

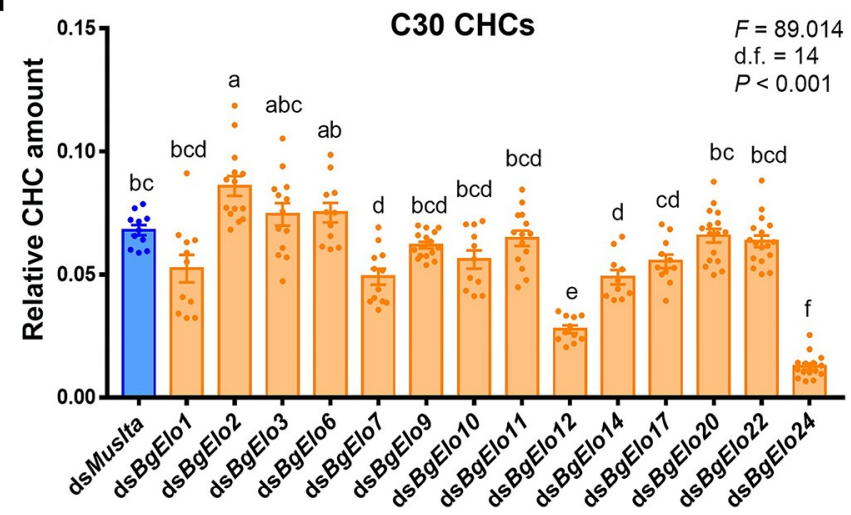

Fig 2. RNAi screen to identify BgElo genes involved in long-chain CHC biosynthesis. Transcript levels of different BgElo genes in the abdominal integument (A) and fat body (B). The numbers on the abscissa correspond to BgElo1-24. Data are shown as mean \pm SEM and calculated from 4 replicates (each replicate contains 4 cockroaches for the integument and 8 cockroaches for the fat body). The influence of knockdown of different $B g E l o$ genes on C27 (C), C28 (D), C29 (E), and C30 (F) CHCs is shown. Data are presented as mean \pm SEM and calculated from 10 to 16 AD2 female cockroaches, each dot indicating a single datum from one cockroach. Only knockdown of BgElo12 and BgElo24 resulted in significant increases of C27 CHCs and decreases of C29 CHCs. Different letters indicate statistically significant differences between groups using Welch ANOVA (Games-Howell multiple comparisons test, $P<0.05)$. The data underlying this figure are included in S1 Data and S2 Table. AD, adult day; CHC, cuticular hydrocarbon; RNAi, RNA interference. 
most striking result was a sharp decline that resulted from BgElo24-RNAi (S3 Fig). The detailed changes of individual CHCs after knockdown of BgElos are available in S2 Table. In conclusion, these data strongly suggest that BgElo12 and BgElo24 are involved in CHC production, but we cannot rule out that other BgElo genes might have a less prominent role in $\mathrm{C} 27$ to C30 HC biosynthesis.

\section{BgElo12 is the terminal gene in maintaining SDHC profiles}

Although it appears that both BgElo12 and BgElo24 are involved in HC production, it is unclear which gene is responsible for sexual dimorphism of CHCs. Therefore, we compared the $\mathrm{CHC}$ profiles of treated female cockroaches with wild-type male cockroaches. The results showed that only RNAi of BgElo12 made the female CHC profile more similar to the male profile (Fig 3A), reflected by a significant increase of $\mathrm{C} 27 \mathrm{CHCs}$ without affecting the maleenriched peak 17, and a selective down-regulation of $\mathrm{C} 29 \mathrm{CHCs}$, with a dramatic down-regulation of the female-enriched peak 24 and some other C29 CHCs (Fig 3B). Also, knockdown of BgElo12 increased the proportions of C27 CHCs and peak 17, while the proportions of C29 CHCs and peak 24 significantly decreased (Fig 3D). These changes converged the female CHC profile toward the male $\mathrm{CHC}$ profile. The repression of BgElo24 down-regulated all C28 to C32 CHCs (Fig 3C), even though knockdown of BgElo24 increased the proportion of C27 CHCs and decreased the proportion of $\mathrm{C} 29$ CHCs and peak 24 (Fig 3E); the amount of the male-enriched peak 17 was also dramatically down-regulated (Fig 3C). Similar results were generated in males, and RNAi of BgElo12 generated the characteristics of male $\mathrm{CHC}$ profiles (S4A Fig), while BgElo24-RNAi down-regulated all C28 to C32 CHCs (S4B Fig). In order to confirm these results, a second RNAi target was used for both genes, and similar results were generated (S5A and S5B Fig). In addition, we analyzed the internal HCs after repression of BgElo12 or BgElo24. The internal HCs underwent similar changes as CHCs (S5C and S5D Fig). These results suggest that the changes imposed by BgElo12- or BgElo24-RNAi were caused by a deficiency in de novo HC biosynthesis and not the transport of HCs from internal tissues to the cuticle.

We also examined the spatiotemporal expression of BgElo12 and BgElo24. Both BgElo12 and BgElo 24 were primarily expressed in the abdominal integument, where the oenocytes that produce $\mathrm{HCs}$ are located (S6 Fig). Monitoring of BgElo12 expression in females and males across different developmental stages showed that female and male BgElo12 mRNA levels were similar at N6D4 (4-day-old sixth-instar nymph) and early adult stage, but its expression level was higher in females than in males at AD3, and the difference increased through AD6 (Fig $3 \mathrm{~F}$ ), a pattern similar to the production of SDCHCs. The expression levels of BgElo24 were higher in males than in females starting at AD2 (Fig 3G). These results also support that only BgElo12, and not BgElo24, is involved in sexual dimorphism of CHCs in B. germanica.

Finally, because CHCs are important waterproofing agents in insects [18], we investigated the roles of BgElo12 and BgElo24 in desiccation resistance. We found that repression of BgElo24 dramatically decreased tolerance of desiccation, but RNAi of BgElo12 had little effect on desiccation tolerance (Fig $3 \mathrm{H}$ ). These results further indicate that the biological significance of BgElo12 in cockroaches may sustain sexual dimorphism of CHCs, whereas BgElo24 may contribute to desiccation tolerance.

\section{BgElo24 provides VLCFA substrates for BgElo12}

Thus far, we showed that both BgElo12 and BgElo24 are involved in HC production: RNAi of BgElo12 only selectively decreased some HCs, while repression of BgElo24 dramatically downregulated all C28 to C32 HCs, and some HCs were affected by both BgElo12 and BgElo24. We 


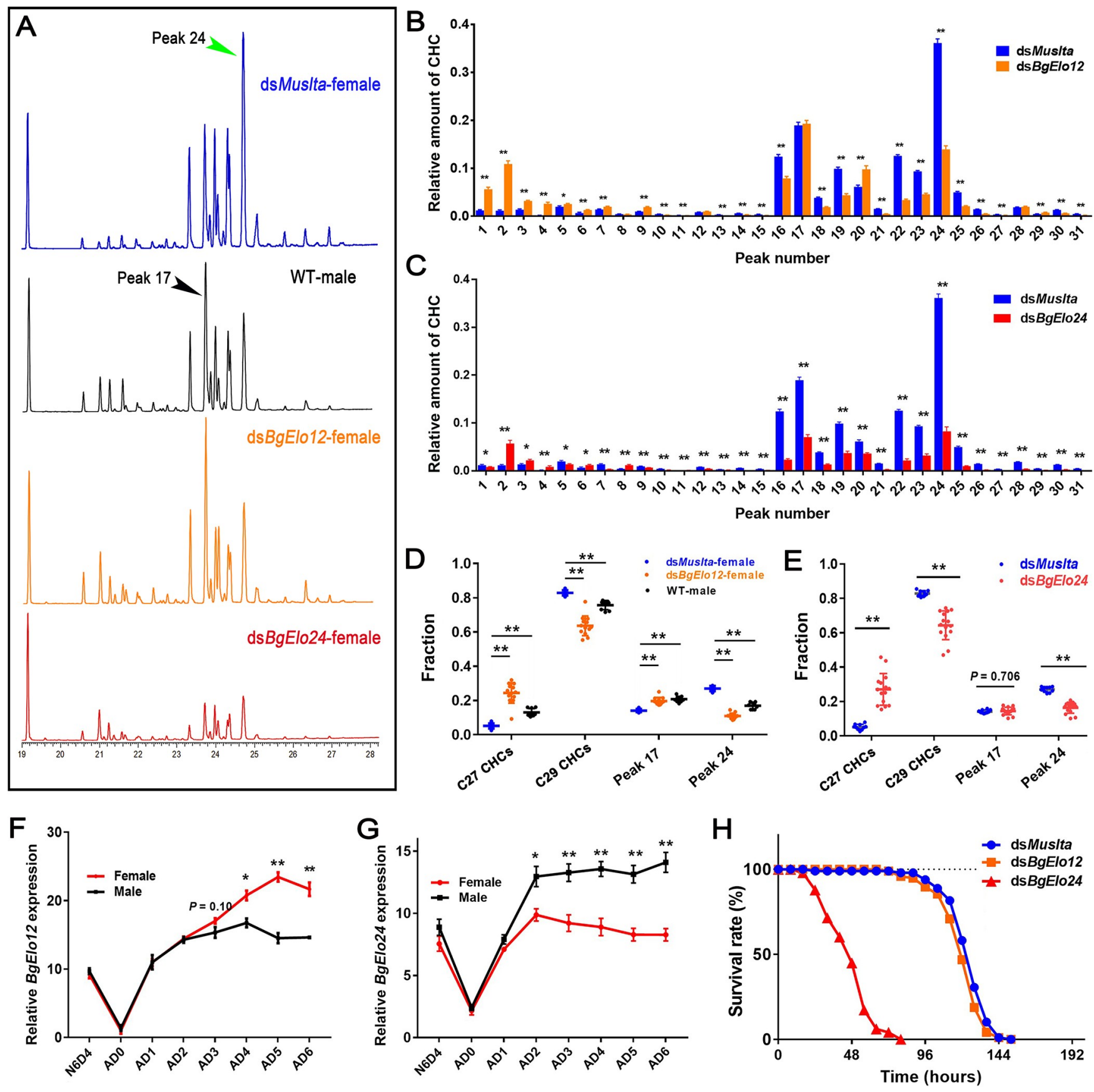

Fig 3. BgElo12 is involved in generating SDHCs. (A) Gas chromatogram of CHC profiles from differently treated cockroaches. The CHC profiles of sexually mature females and males (AD6-AD8) showed a marked difference, and RNAi of only BgElo12 in females generated a male-like CHC profile. Quantitative influence of BgElo12-RNAi (B) and BgElo24-RNAi (C) in females on the amount of each CHC (the peak numbers corresponding to S1 Data). Percentage change of the C27 CHCs, C29 CHCs, male-enriched peak 17, and female-enriched peak 24 after BgElo12-RNAi (D) or BgElo24-RNAi (E). Data in (B-E) are shown as mean $\pm \mathrm{SEM},{ }^{*} P<0.05,{ }^{* *} P<0.01$; differences between 2 groups were determined by 2 -tailed Student $t$ test, and differences among 3 groups were determined by 1-way ANOVA and LSD multiple comparisons, $n=10$ for $d s M u s l t a, 11$ for $d s B g E l o 12,15$ for $d s B g E l o 24$, and 9 for WT male. Relative expression of BgElo12 (F) and BgElo24 (G) among different developmental stages and different ages of adult males and females. $P$ values were calculated from 4 replicates (2-3 cockroaches/replicate), ${ }^{* *} P<0.01 ; 2$-tailed Student $t$ test. (H) Survival rates of $d s M u s l t a(n=98), d s B g E l o 12(n=96)$, and $d s B g E l o 24$ ( $\left.n=98\right)$ treated female cockroaches maintained at $5 \%$ RH. Survival rates were calculated every 8 hours until all cockroaches died. The data underlying this figure are included in S1 Data. CHC, cuticular hydrocarbon; LSD, least significant difference; RH, relative humidity; RNAi, RNA interference; SDHC, sexually dimorphic hydrocarbon; WT, wild-type.

https://doi.org/10.1371/journal.pbio.3001330.g003 
also found that knockdown of BgElo24 caused a 2-fold up-regulation of BgElo12, while RNAi of BgElo12 had no significant influence on BgElo24 mRNA level (S7A and S7B Fig). Therefore, we considered whether BgElo24 is a basic elongase that provides substrates used for HC biosynthesis and if BgElo24 generates primary precursors that can be further catalyzed by BgElo12. We verified the functions of BgElo12 and BgElo24 in VLCFA biosynthesis with heterologous expression of BgElo12 and BgElo24 in Saccharomyces cerevisiae (strain INVSc1). The genetic background of S. cerevisiae is relatively clear, as wild-type $S$. cerevisiae contains 3 different ELO proteins: ELO1 is able to elongate C14 fatty acids (FAs) to C16 FAs, ELO2 can generate C24 FAs, and ELO3 plays essential roles in the conversion of C24 FAs to C26 FAs [59,60]. In our study, activation of GAL1 promoter by galactose successfully transcribed the target genes, and we detected the expression of GFP protein, which suggests that this expression system is effective (S7C-S7E Fig). GC-MS analysis of FAs in S. cerevisiae that contained pYES2-GFP (control) detected large amounts of C16:1, C16:0, C18:1, and C18:0 FAs, minor amounts of C20 to C24 and C28 FAs, and a larger amount of C26:0 FAs (S3 Table). Yeast with exogenous BgElo12 did not produce any new FAs, but heterologous expression of BgElo24 sharply increased the amount of C28:0 FA and generated a new component, C30:0 FA (Fig 4 $\mathrm{A}-\mathrm{C}, \mathrm{S} 7 \mathrm{H}$ and S7I Fig). These results suggest that BgElo24 is capable of elongating endogenous yeast FAs to generate C28:0 and C30:0 FAs.

Considering that BgElo12 and BgElo24 may selectively elongate substrates with specific carbon chain lengths, we separately added C20:0, C22:0, C24:0, C26:0, and C28:0 FAs to the medium. Compared with the control, BgElo24 did not produce any new FAs when C20:0, C22:0, C24:0, or C26:0 were added (S3 Table). However, when C28:0 FA was added, yeast with pYES2-BgElo12 generated C30:0 FA, although in small amounts (Fig 4A'-4C', S3 Table). These results suggest that BgElo24 not only directly provides substrates (C28 and C30 fatty acyl-CoAs) for the biosynthesis of C27 and C29 $n$-alkanes, but also provides C28 fatty acylCoA for BgElo12 to elongate to C30 fatty acyl-CoA, which, in turn, generates C29 n-alkane. This might be the reason why in vivo RNAi of BgElo12 slightly decreased C29 $n$-alkane and caused a dramatic increase in C27 $n$-alkane, whereas RNAi of BgElo24 dramatically decreased C29 n-alkane.

In order to analyze the activity of BgElo12 and BgElo24 in elongating methyl-branched FAs, 2 representative substrates, 2-MeC16:0 and 14-MeC16:0 FAs, the potential substrates for 15-methyl HCs and 3-methyl HCs, respectively, were added to the medium. However, we found that these substrates could not be catalyzed by BgElo12 or BgElo24 (S7F-S7F" and S7G-S7G" Fig). We suspect that this might be caused by a shortcoming of the yeast FA elongation system. FA elongation requires an elongase and 3 other enzymes [29]. The last 3 yeast endogenous enzymes may not catalyze methyl-branched substrates. In conclusion, these results indicate that both BgElo12 and BgElo24 catalyzed the biosynthesis of VLCFAs in the yeast heterologous system, and BgElo24 was able to provide primary substrates for BgElo12. However, the activity of BgElo12 and BgElo24 in methyl-branched FA elongation needs further investigation.

\section{Female-enriched HCs are crucial for contact sex pheromone-based courtship performance}

The largest single chromatographic HC peak in females contains 3,7-; 3,9-; and 3,11-DimeC29, which is much less represented in males. Because 3,11-DimeC29 has been shown to be the precursor for the contact sex pheromone 3,11-DimeC29-2-one (C29 methyl ketone) in B. germanica [48], we suspected that the female-specific HC profile may be important for maintaining a high level of contact sex pheromone. We first monitored the pattern of 3,11-DimeC29-2-one accumulation in the first gonotrophic cycle. The contact sex pheromone 


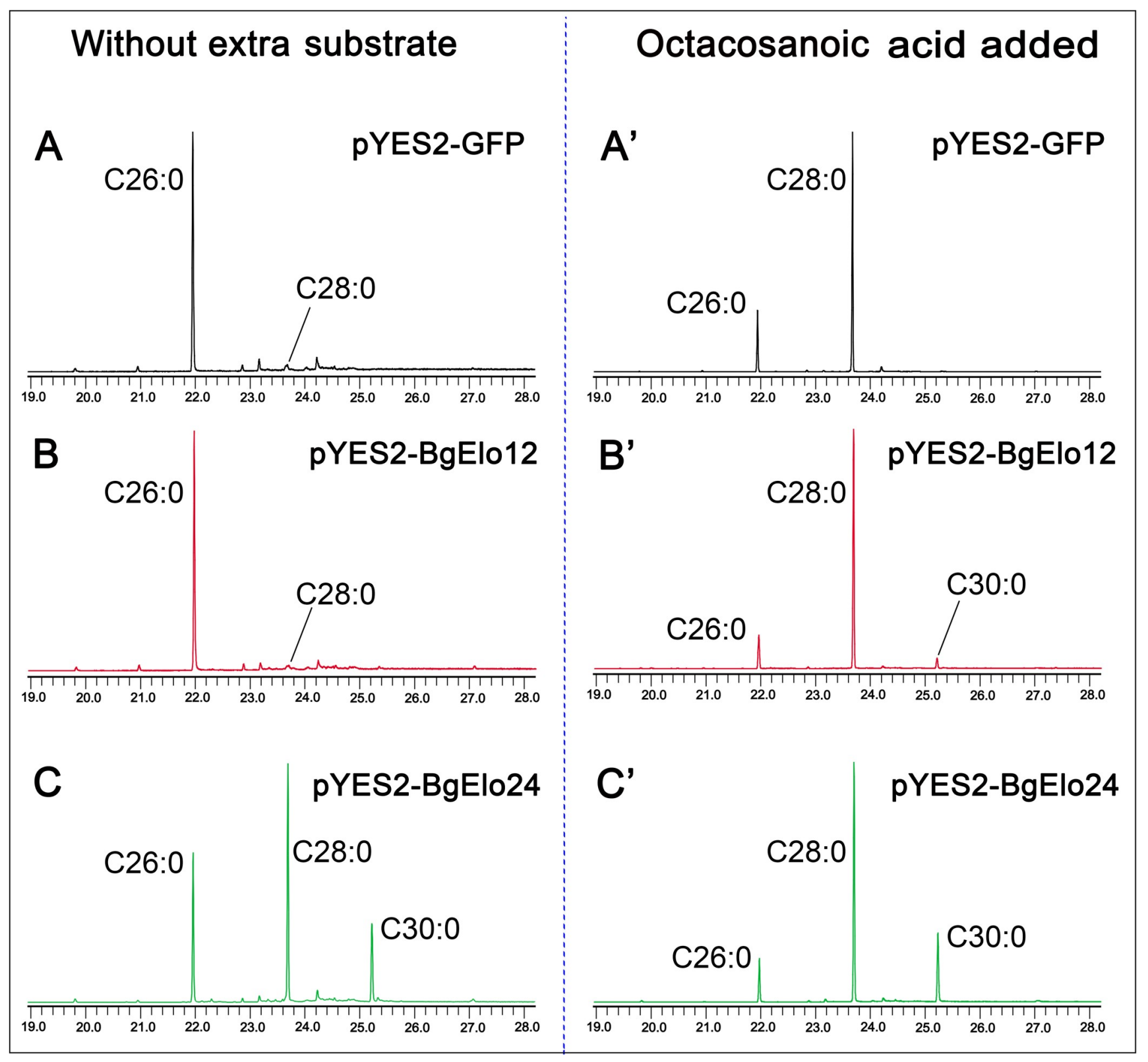

Fig 4. Yeast expression and substrate catalysis of BgElo12 and BgElo24. Representative gas chromatogram of FAMEs of yeast transformed with pYES2-GFP (A), pYES2-BgElo12 (B), and pYES2-BgElo24 (C) without addition of extra substrates. After adding octacosanoic acid (C28:0), the corresponding chromatograms are shown at the right for pYES2-GFP (A'), pYES2-BgElo12 (B'), and pYES2-BgElo24 (C'). The chromatograms are truncated, and only chromatographic peaks of interest are indicated. The FAMEs are labeled by their corresponding saturated FAs. Complete chromatograms are shown in S7 Fig. The data underlying this figure are included in S3 Table. FA, fatty acid; FAME, fatty acid methyl ester.

https://doi.org/10.1371/journal.pbio.3001330.g004

showed a stable low level at the early adult stage (AD0 to AD2), its accumulation started at AD3, quickly increased from AD5 to AD7 (Fig 5A), but decreased at AD8 when most females oviposited. Analysis of the influence of BgElo12-RNAi on C29 methyl ketone was performed at AD6. Female cockroaches were subjected to 3 consecutive dsRNA injections before being subjected to lipid analysis: the first one at early fifth instar, the second at early sixth instar, and the third at AD1. We found that repression of BgElo12 reduced the C29 methyl ketone by more than $75 \%$ in both cuticular and internal extractions (Fig 5B-5D). 

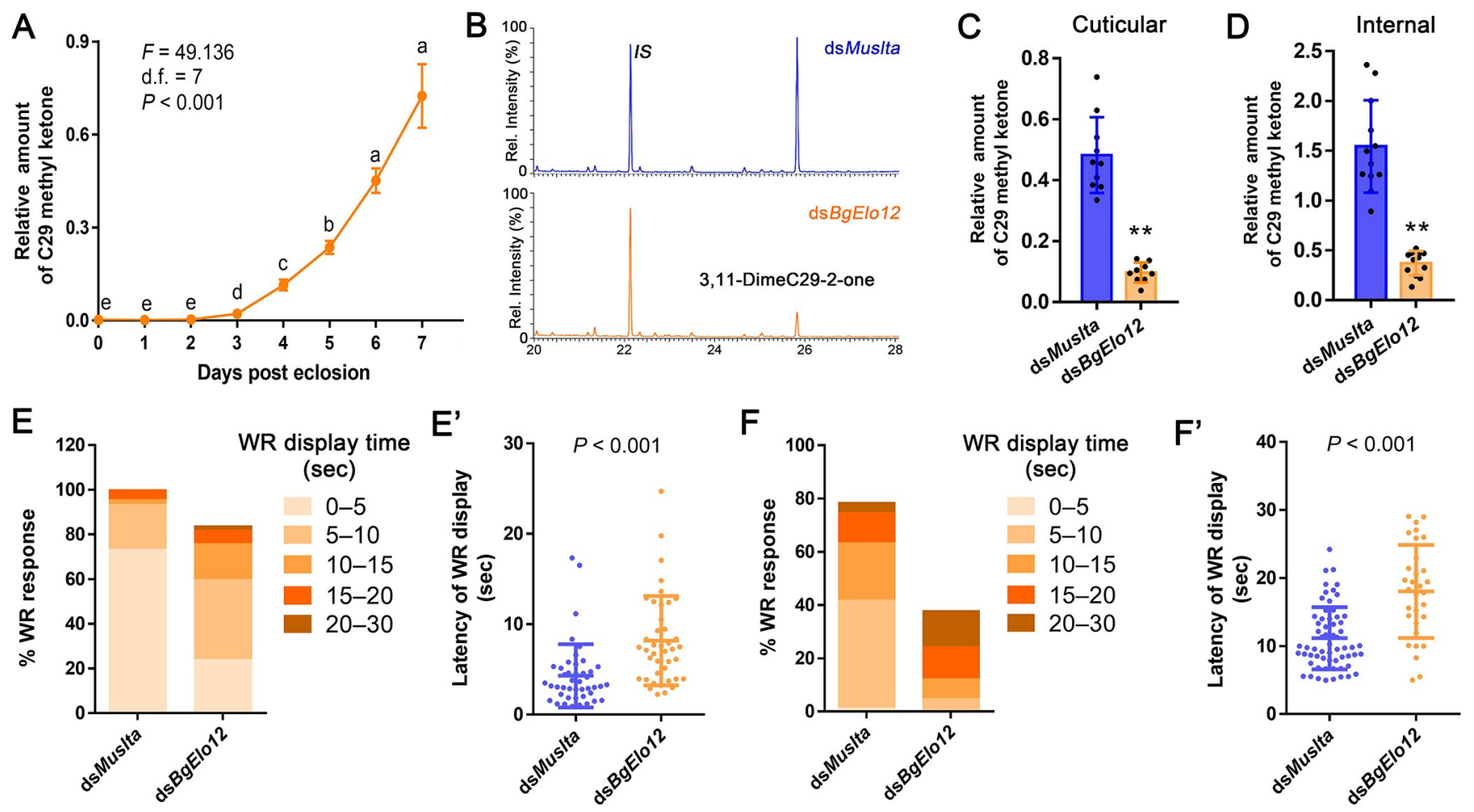

Fig 5. BgElo12-RNAi effects on contact sex pheromone biosynthesis in females and male courtship performance. (A) The pattern of contact sex pheromone (3,11-DimeC29-2-one) accumulation during female sexual maturation. Data are shown as mean \pm SEM. Different letters indicate significant differences between groups using Welch ANOVA (Games-Howell multiple comparisons test, $P<0.05$ ). (B) Representative chromatogram showing lower amounts of sex pheromone after BgElo12-RNAi. IS is the internal standard 14-heptacosanone. Relative cuticular (C) and internal (D) amounts of 3,11-DimeC29-2-one (C29 methyl ketone) after BgElo12-RNAi. Data are shown as mean $\pm \mathrm{SD}$, and each replicate is shown as a dot. ${ }^{* *} P<0.01,2$-tailed Student $t$ test; $n=9-12$. Influence of BgElo12-RNAi on the ability of AD5 (E-E') and AD3 (F-F') female antennae to elicit courtship in males. The percentage of males that responded with a WR behavior in response to contact with female antenna were determined for 44 ( $d s M u s l t a)$ and 49 (dsBgElo12) female antennae in (E) and 79 (dsMuslta) and $82(\mathrm{dsBgElo12})$ antennae in (F). Each female antenna was tested only once with a single male. The proportion of WR display over different time periods (latency, seconds) is shown in progressively darker colors. (E') and (F') Average latency of WR display calculated from those antennae that successfully activated a courtship behavior of males within 30 seconds. Data are shown as mean \pm SD; each dot represents a datum calculated from one antenna. $P$ values were determined by 2 -tailed Student $t$ test. The data underlying this figure are included in S1 Data. AD, adult day; RNAi, RNA interference; WR, wing raising.

https://doi.org/10.1371/journal.pbio.3001330.g005

We next determined whether the BgElo12-RNAi repression of pheromone production in females affected male courtship behavior. When the female contact sex pheromone is detected by a male, it displays a characteristic male courtship behavior, including wing raising (WR) [50]. When an antenna of AD5 female was used as stimulus, nearly all males responded. RNAi of BgElo12 resulted in only a slight decline in WR rate (about 20\%), but the latency of WR was significantly increased (Fig 5E and 5E'). For using the antenna of AD3 females, we found that males responded to $80 \%$ of the control antennae with the WR display, but less than $40 \%$ of the antennae from BgElo12-RNAi females elicited WR in males. The average latency of WR toward antennae of BgElo12-RNAi females was 18.03 seconds, while the control antennae elicited WR in 11.05 seconds (Fig 5F and 5F'). These results indicate that female-enriched HCs are advantageous for contact sex pheromone biosynthesis, especially at the early sexual maturation stage.

\section{Sex differentiation genes modulate BgElo12}

The female-enriched HC composition is tightly associated with a higher expression of BgElo12 in females, but the regulators that govern sexually dimorphic expression of BgElo12 in females and males are unknown. Wexler and colleagues reported that RNAi of the sex determination 
gene BgTra in females converts its CHC profile to a male-like profile [52]. Taken together with our results, it would appear that the sex differentiation pathway might be involved in regulating BgElo12 expression. We first confirmed the function of BgTra and BgDsx in sex-specific development via RNAi. For each gene, we used 2 unique RNAi targets within a conserved sequence region for multiple isoforms. RNAi of BgTra and $B g D s x$ in females or males showed similar phenotypes as described by Wexler and colleagues [52]. BgTra is only functional in females, while $B g D s x$ only works in males (S8 Fig). We next studied the expression of BgTra and $B g D s x$ in females and males using quantitative PCR (qPCR) primers that target all isoforms of each gene. The expression of both $B g T r a$ and $B g D s x$ increased after eclosion, but $B g T r a$ transcript levels tended to be stable from AD4 to AD6 (Fig 6A and 6B). RNAi of BgTra
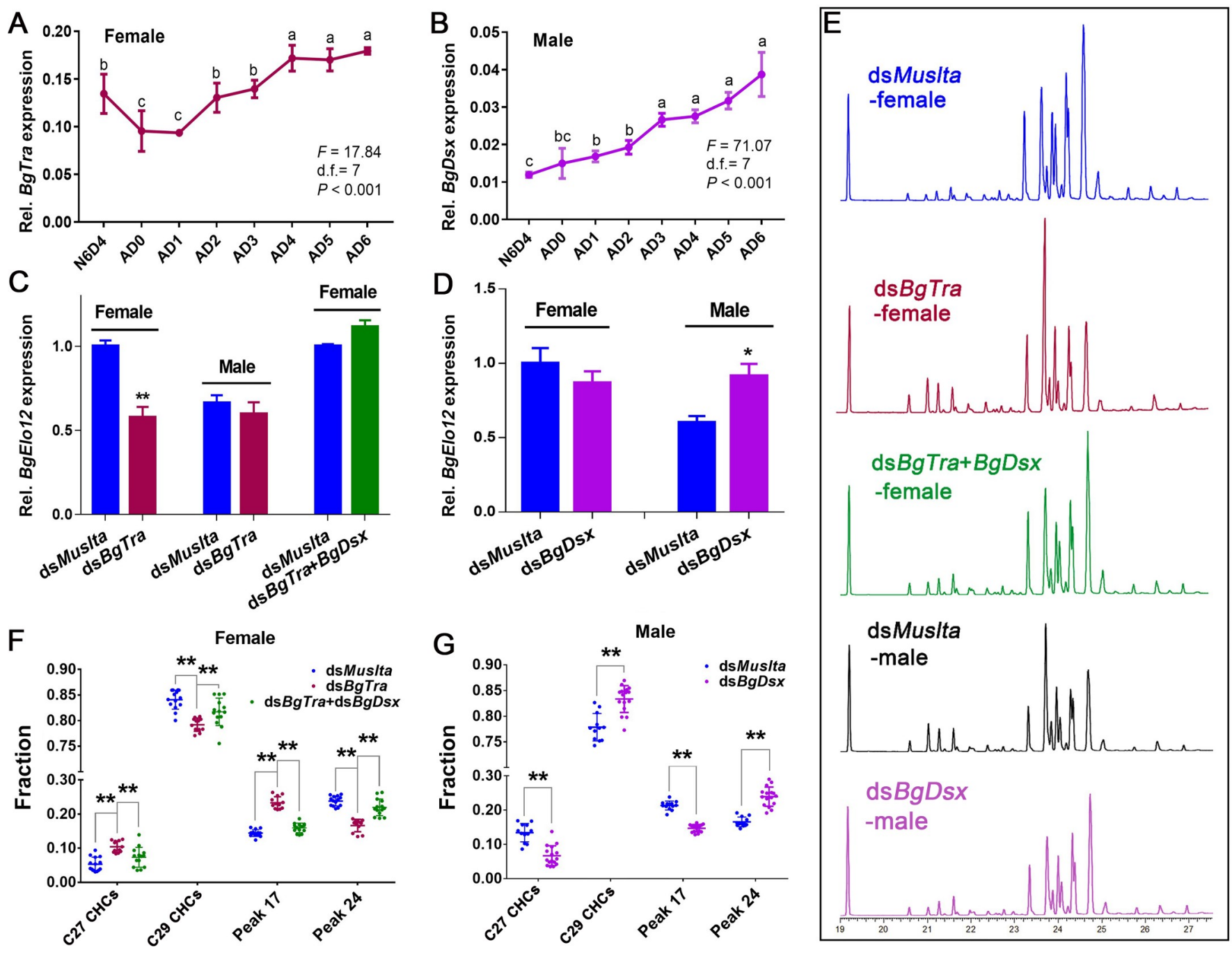

Fig 6. Regulation of BgElo12 and SDHC profiles by sex differentiation genes. Temporal patterns of BgTra expression in females (A) and BgDsx expression in males (B). Data are shown as mean \pm SEM and calculated from 4 replicates (2-3 cockroaches/replicate). N6D4 is sixth-instar nymph on day 4, AD0-AD6 represent adult days 0 to 6 . Different letters indicate significant differences between the groups (ANOVA, Fisher LSD, $P<0.05$ ). (C, D) Regulation of $B g E l o 12$ expression by BgTra and BgDsx. Data are shown as mean calculated from 4 replicates $(2-3$ cockroaches/replicate $) \pm$ SEM; ${ }^{*} P<0.05$, ${ }^{*} P<0.01,2$-tailed Student $t$ test. (E) Gas chromatograms of CHCs after repressing different sex differentiation genes. (F) Regulation of the proportions of representative CHCs by BgTra in females. Data are presented as mean $\pm \mathrm{SD},{ }^{*} P<0.01$; differences between 2 groups were determined by 2 -tailed Student $t$ test, differences among 3 groups were determined by 1-way ANOVA and LSD multiple comparisons; $n=14$ for $d s M u s l t a, 12$ for dsBgTra, and 14 for dsBgTra+dsBgDsx. (G) The proportion change of representative CHCs after BgDsx-RNAi in males. Data are shown as mean $\pm \mathrm{SD}$; ${ }^{* *} P<0.01$; 2-tailed Student $t$ test, $n=12$ for $d s M u s l t a$ and 16 for $\mathrm{dsBgDs} x$. The data underlying this figure are included in S1 Data. AD, adult day; CHC, cuticular hydrocarbon; LSD, least significant difference; RNAi, RNA interference; SDHC, sexually dimorphic hydrocarbon. 
in females significantly decreased the mRNA level of BgElo12, but BgElo12 expression was unaffected in males (Fig 6C). Notably, the down-regulation of BgElo12 by dsBgTra injection in females could be rescued by co-injection of dsBgTra and dsBgDsx (Fig 6C), but ds $B g D s x$ alone was not functional in regulating BgElo12 expression in females (Fig 6D). Conversely, in males, knockdown of BgDsx alone increased the expression of BgElo12 (Fig 6D). As RNAi of BgTra in females generated the male type $B g D s x\left(B g D s x^{M}\right)$ [52], we suspect that the down-regulation of $B g E l o 12$ in females after RNAi of $B g T r a$ was caused by the generation of $B g D s x^{M}$. This hypothesis was supported by co-injection of dsBgTra and ds $B g D s x$ and a dual-luciferase reporter assay that $\mathrm{BgDsx}^{\mathrm{M}}$ could directly modulate the expression of $B g E l o 12$ by interaction with its upstream sequence region (S9 Fig). Further, we found that RNAi of neither BgTra in females nor BgDsx in males could affect the expression of BgElo24 (S10 Fig), suggesting that BgElo24 is not regulated by these 2 sex differentiation genes.

We further analyzed the effects of $B g T r a$ and $B g D s x$ on HC profiles. Knockdown of $B g T r a$ in females masculinized the $\mathrm{CHC}$ profile and increased the male-enriched $\mathrm{CHC}$ components; however, this change disappeared by co-injection of dsBgTra and dsBgDsx (Figs 6E and 6F, S11A). Similarly, repression of $B g D s x$ in males feminized the $\mathrm{CHC}$ profile and increased the female-enriched components (Figs $6 \mathrm{E}$ and $6 \mathrm{G}, \mathrm{S} 11 \mathrm{~B}$ ). In order to verify that the sex differentiation genes affected $\mathrm{CHC}$ profiles by regulating the generation of HCs but not their selective transport and deposition to the cuticle, we analyzed the internal HCs. RNAi of both BgTra in females and $B g D s x$ in males caused an intersexual conversion of internal HC profiles, as seen in the CHCs (S12A-S12D Fig). Overall, our results indicate that the sexual dimorphism of HCs in B. germanica is primarily determined by BgElo12, and sex determination pathway genes are the critical regulators that control the asymmetric expression of BgElo12 between males and females.

\section{Discussion}

Our study reveals a novel molecular mechanism responsible for the formation of SDHCs in $B$. germanica. The $\mathrm{CHC}$ profiles in insects are regulated by complex biosynthetic and transport pathways, involving multiple gene families. We demonstrated that the fatty acid elongation step is responsible for sexual dimorphism of CHCs in B. germanica, We identified that both BgElo12 and BgElo24 were participated in HC biosynthesis, but only the female-enriched $B g E l o 12$ is the core gene that encodes for the elongase involved in generating more femaleenriched HCs, and the asymmetric expression of BgElo12 between the sexes is modulated by sex differentiation genes: $B g D s x^{M}$ represses the expression of $B g E l o 12$ in males, while $B g T r a$ removes this repression in females (Fig 7). Because a female-enriched HC serves as a precursor to a female contact sex pheromone, we also revealed the prominence of BgElo12 in sexual behavior.

\section{Fatty acid chain elongation is a key step in the regulation of SDHCs in $B$. germanica}

The diversity of HCs in insects is reflected in the HC carbon chain lengths, their degree of saturation, and the number and positions of methyl groups [39]. HCs in B. germanica are composed of only $n$-alkanes and methyl-branched alkanes, and the fatty acid biosynthesis gene that governs the incorporation of methyl groups in the aliphatic chain showed no role in generating the sexual dimorphism of HCs in B. germanica [55]. In this study, we found that sexually mature female cockroaches contain relatively higher amounts of C29 HCs than males, whereas male cockroaches had more C27 HCs, suggesting that chain length is an important factor in the dimorphism of HCs. 


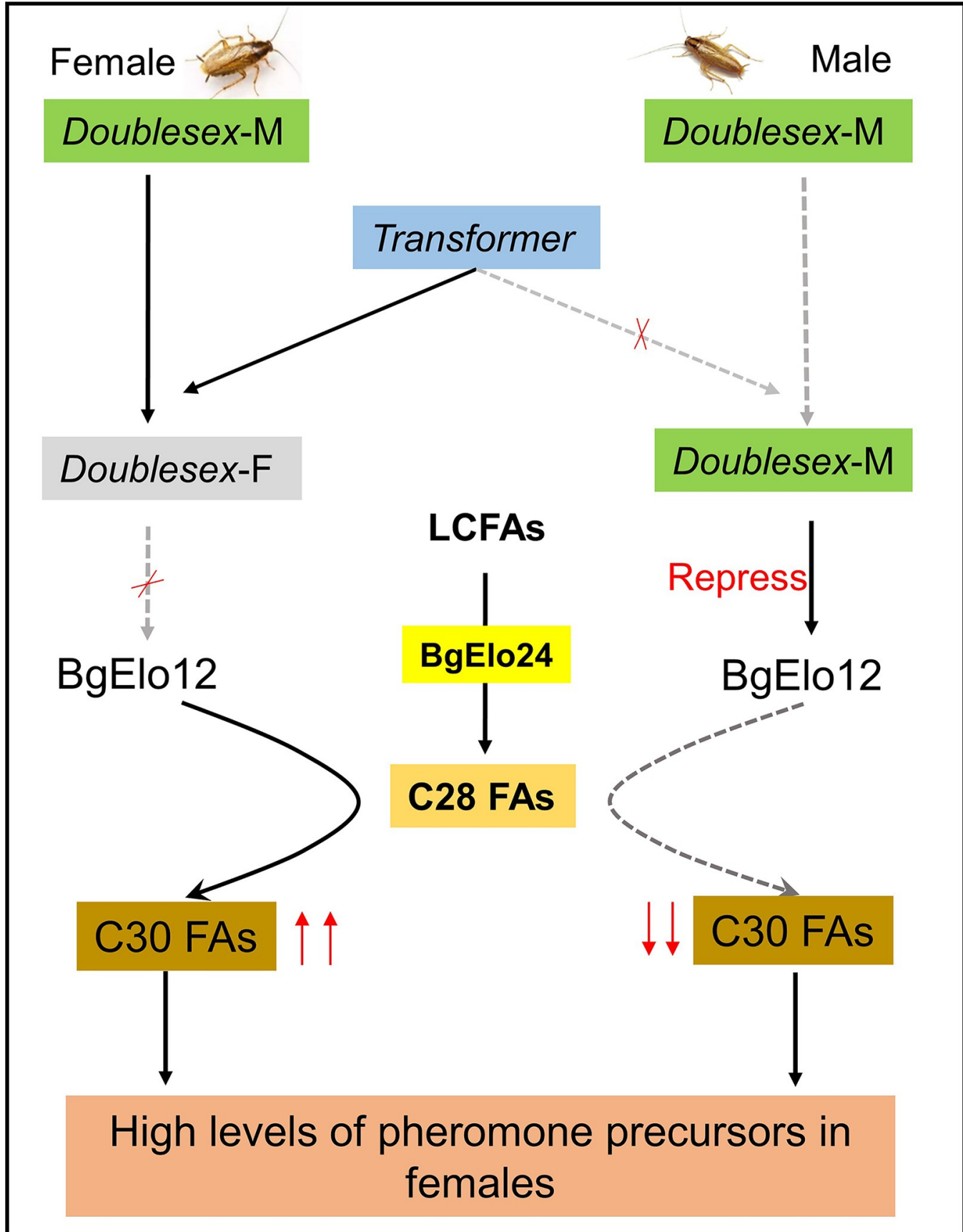

Fig 7. Model for BgElo12 in generation SDHCs in B. germanica. Both BgElo12 and BgElo24 participated in HC biosynthesis, but BgElo24 is a basic fatty acid elongase that is able to catalyze a wide range of substrates and provides $\mathrm{C} 28$ FAs for BgElo12 to generate specific C30 FAs. The male-specific Doublesex (Doublesex-M) is able to repress the transcription of BgElo12 in males. However, Transformer only functions in females and can splice Doublesex-M to the nonfunctional female type Doublesex (Doublesex-F); thus, BgElo12 is highly expressed in females and generates more C30 FAs. A high level of C30 FAs lastly produces more contact sex pheromone precursors in females. FA, fatty acid; HC, hydrocarbon; LCFA, long-chain fatty acid; SDHC, sexually dimorphic hydrocarbon.

https://doi.org/10.1371/journal.pbio.3001330.g007

Studies of the genetic bases of HC biosynthesis in insects have demonstrated that chain lengths are determined by the fatty acid elongation process: The rate-limiting enzyme elongase determines the chain lengths of VLCFA products and produces the final HCs with different 
chain lengths [30]. In our study, an RNAi screen identified that BgElo12 and BgElo24 were involved in HC biosynthesis. Expression of both genes in yeast demonstrated that both BgElo12 and BgElo24 displayed functions similar to ELOVL4 in mammals, which showed a special function in the biosynthesis of VLCFAs with chain lengths greater than C28 [61]. BgElo24, however, is a more fundamental elongase that seems able to catalyze a wide range of substrates to generate FAs of various chain lengths and can also provide primary substrates for BgElo12 to produce C30 FAs. Notably, only BgElo12 had higher expression in females, which was consistent with the higher amounts of C29 HCs in females. Knockdown of BgElo12 in females caused a dramatic decrease of the female-enriched peak $24(3,7-; 3,9-;$ and 3,11-DimeC29), but it did not affect the male-enriched peak 17 (9-; 11-; 13-; and 15-MeC29), which generated a male-like HC profile; however, RNAi of BgElo24 unselectively down-regulated all C28 to C30 HCs, indicating that only BgElo12 was involved in the formation of SDHCs in B. germanica. Lastly, we found both RNAi of BgElo12 and BgElo24 increased some $\mathrm{C} 27 \mathrm{HCs}$, indicating that there are some other BgElos involved in HC production. The identification of these genes will be arduous, because the $\mathrm{C} 27$ compounds occur in small amounts, and multiple BgElos may catalyze the synthesis of the same HC independently. It is worth noting in this regard that Blattella asahinai, a sister species of B. germanica produces almost no C27 compounds [62]. These 2 species can hybridize, potentially offering a resource for the genetic regulation of $\mathrm{C} 27 \mathrm{HCs}$.

In B. germanica, as in other insects, HCs are transported through the hemolymph and selectively incorporated into or deposited on various tissues, including the cuticle, ovaries, and specialized pheromone glands $[47,63,64]$. In our study, analysis of the influence of BgElo12-RNAi on internal HCs demonstrated that the female- and male-specific $\mathrm{CHC}$ profiles were not caused by selective transport, but rather by differences in de novo $\mathrm{HC}$ biosynthesis between the sexes, regulated by BgElo12. Also, the total amount of internal HCs was significantly decreased in BgTra-RNAi females, while it was increased in BgDsx-RNAi males (S12E and S12F Fig). These results suggest that there is an unusual transport of HCs after repression of $B g T a r$ or $B g D s x$. We suspect that the unusual transport of HCs may be caused by changes in the capacity to store internal HCs, as large amounts of internal HCs are shunted to the ovaries $[45,65]$, and sex differentiation genes are the key regulators of normal ovary development in females and repression of ovary generation in males $[8,52]$. We conclude that although the transport of HCs from internal tissues to the cuticle was affected after repressing BgDsx or $B g T r a$, the effect was on overall HC transport, with no apparent selective transport on specific HCs. The results suggest that the influences of sex differentiation genes on SDHCs are not caused by selective transport, but may largely relay on the regulation of BgElo12 expression. Elongases have been shown to participate in HC production in several other insect species including D. melanogaster, Nilaparvata lugens, and Locusta migratoria [33,66,67]. The elongase gene eloF was also shown to be specifically expressed in females and may be regulated by the sex differentiation gene Transformer, but the specific details are not very clear [33]. Nevertheless, the roles of elongase genes in sexual dimorphism of HCs in other insects have not been described.

\section{BgElo12 and $B g D s x^{M}$ are key nodes connecting the HC synthesis and sex differentiation pathways}

In this study, we first demonstrated that BgElo12 is the key regulator in the HC biosynthesis pathway, responsible for the differences in the HC profiles between females and males. We next explored the upstream regulators that modulate the sexually dimorphic expression of BgElo12. The molecular genetic switches that determine which sex determination pathway is 
followed by males and females are highly variable in animals. The doublesex/mab-3 related $(D m r t)$ family of transcription factors includes conserved developmental regulators in the sex differentiation pathway, governing the fate of sexually dimorphic traits in animals [8]. Dsx in arthropods, which is related to Dmrt, works through sex-specific splice variants that are controlled by Tra in many insects. Sex-specific $D s x$ isoforms promote sexual differentiation by modulating diverse downstream genes; thus, the $D s x$ gene is regarded as a central nexus in sexual differentiation $[68,69]$. Previous work reported that knockdown of BgTra converted the female cockroach CHC profiles to male-like profiles [52]. In our study, RNAi of BgTra in female cockroaches indeed down-regulated the expression of BgElo12, but the effect of BgTraRNAi on BgElo12 expression could be recovered by co-injection of dsBgTra and dsBgDsx, and RNAi of $B g D s x$ in males up-regulated BgElo12 transcripts. These results suggest that $B g D s x^{M}$ can repress the transcription of BgElo12 in males, and the down-regulation of BgElo12 by repressing $B g T r a$ was caused by the conversion of $B g D s x^{F}$ to $B g D s x^{M}$, as $B g T r a$ is able to regulate the splicing of $B g D s x^{M}$ to $B g D s x^{F}$ [52]. The regulation of $B g E l o 12$ by $B g D s x$ connects the sex differentiation pathway with the HC biosynthesis pathway, and, therefore, enables the sexual dimorphism of HCs in B. germanica.

Although the dual-luciferase reporter gene assay suggested that $B g D s x^{M}$ can directly regulate the transcript of BgElo12, there may be other indirect regulatory pathways. Several candidate factors have been shown to regulate the HC profiles in various insects (e.g., D. melanogaster and Musca domestica), including ecdysone, juvenile hormone (JH), biogenic amines, and the insulin signaling pathway [26,70-77]. It has been reported that ecdysone is able to regulate the fatty acyl-CoA elongation step and shift the chain lengths of HCs in M. domestica [26]. Ecdysone is mainly produced by the prothoracic gland, but, in some insects, also by the ovaries $[78,79]$, and the development of the ovaries is under the regulation of the sex differentiation pathway. Moreover, endocrine signals like $\mathrm{JH}$ and insulin are also regulated by the sex differentiation pathway [80]. Therefore, these signals may be potential mediators that complete the regulatory network between $B g D s x$ and BgElo12. However, more detailed investigations are needed to thoroughly elucidate the differential regulation of HC production in both females and males.

$D s x$ also regulates the HC biosynthesis pathway in D. melanogaster; $D s x^{F}$ specifically activates the transcription of desat $F$ and generates pheromonal dialkenes [42]. However, $B g D s x^{M}$ suppresses the generation of female traits in male cockroaches, while BgTra removes the inhibitory effect of $B g D s x^{M}$ in females [52]. Thus, $B g D s x^{M}$ represses the expression of BgElo12, and, therefore, it represses the generation of female-enriched HCs in males, and BgTra is crucial in maintaining female-enriched HCs, especially for the contact sex pheromone precursors. However, BgTra may also affect contact sex pheromone biosynthesis in female cockroaches through additional pathways.

\section{Biological significance of the sexual dimorphism of HCs}

Sexually dimorphic traits are generated in females and males in response to intra- and intersexual selection, but pleiotropic traits are also subject to natural selection, especially when they are also shaped by and adapted to environmental stresses [3,81]. Most moth sex pheromones are $\mathrm{C} 12$ to $\mathrm{C} 18$ aldehydes, alcohols, and acetate esters, derived from fatty acids in specialized pheromone glands. These pheromones appear to have no other function beyond attracting the opposite sex. In contrast, $\mathrm{CHC}$ pheromones appear to serve both in sexual communication and in waterproofing of the cuticle. The female-specific contact sex pheromone of B. germanica is clearly subject to both natural and sexual selection because it is derived from a prominent $\mathrm{CHC}$, which also serves as a waterproofing component of the $\mathrm{CHC}$ profile and is maternally invested in offspring [82]. Thus, maintenance of HC contact sex pheromone 
precursors in female cockroaches is adaptive in both reproductive success and survival in an arid environment. The quality of insect pheromones is considered an honest indicator of fitness potential [73]. This assertion may be particularly pertinent in B. germanica, where the pheromone and its HC precursor serve in sexual communication, resilience to environmental stressors, and in maternal investment in eggs.

In systems that use $\mathrm{CHCs}$ in sexual communication, it is common for the $\mathrm{CHC}$ profiles to contain female- and male-specific components, as is evident in Drosophila. In B. germanica, as well, male cockroaches have a unique $\mathrm{CHC}$ profile, especially enriched in $\mathrm{C} 27$ components with 9-; 11-; 13-; and 15-MeC29 being particularly prominent. The male-specific HC profile is generated during sexual maturation, suggesting that it may function in sexual communication. It is possible that the male-enriched 9-; 11-; 13-; and 15-MeC29 may function as a sex pheromone in 3 related contexts: (a) it may distinguish males and females within cockroach aggregations; (b) it may signal "maleness" and male quality to females; and (c) it may function in male-male recognition, contests, and competition for access to females. Moreover, it is possible that male-specific P450s may catalyze the oxidation of these male-enriched HCs to homologous methyl ketones, as in females, and, in turn, serve these functions. However, more bioassays are required to analyze the biological significance of male-specific $\mathrm{CHC}$ profiles.

\section{Materials and methods}

\section{Insect rearing}

The German cockroach B. germanica originated from a laboratory strain collected in the 1970s. The cockroaches were maintained in aquaria at $30 \pm 1^{\circ} \mathrm{C}$ with a relative humidity $(\mathrm{RH})$ of approximately $50 \%$ under 12:12 hour light-dark photoperiod regime and fed rat chow and tap water. Newly hatched cockroaches were separated and reared in new containers. Earlystage (day 1 or 2) fourth- and fifth-instar nymphs were separated and used in dsRNA injection; newly emerged adults were collected and reared in plastic jars for experiments.

\section{Preparation of HCs and methyl ketones}

B. germanica cuticular lipids were extracted as described [55]. Individual adult female cockroaches were sacrificed by freezing at $-20^{\circ} \mathrm{C}$, thawed at room temperature, the cuticle surfaceextracted in $1 \mathrm{~mL}$ of hexane twice, and finally rinsed in $1 \mathrm{~mL}$ of hexane. $n$-Hexacosane $(15 \mu \mathrm{g})$ or 14-heptacosanone $(0.5 \mu \mathrm{g})$ were added as internal standards. The extracts were combined and reduced to approximately $300 \mu \mathrm{L}$ with a nitrogen flow and loaded onto a Pasteur pipette silica gel mini-column, as previously described [55]. The CHCs were eluted with $8 \mathrm{~mL}$ of hexane, and the contact sex pheromone fraction was subsequently eluted with $8 \mathrm{~mL}$ of $3 \%$ ethyl ether in hexane. Internal lipids were extracted from the cockroach following a procedure described by Fan and colleagues [83]. Each surface-extracted cockroach was homogenized in a solution of hexane-methanol-ddH2O (2:1:1 mL), and $30 \mu \mathrm{g}$ of $n$-hexacosane and $1 \mu \mathrm{g}$ of 14-heptacosanone were added for quantification of internal HCs and methyl ketones, respectively. The homogenate was vigorously vortexed and centrifuged at 2,500 $\mathrm{g}$ for 10 minutes. The supernatant hexane phase was collected, and the extraction was repeated using $n$-hexane. Separation of HCs and methyl ketones was performed using column chromatography, as described above.

\section{GC-MS analysis}

Lipid analysis was performed with a TRACE 1310 GC-ISQ single quadrupole MS (Thermo Fisher Scientific, Waltham, Massachusetts, United States of America). In brief, lipids were separated on a DB-5MS capillary column (30-m length, $0.25-\mathrm{mm}$ ID, and $0.25-\mu \mathrm{m}$ film thickness; 
Agilent Technologies, Santa Clara, California, USA). The oven started at $60^{\circ} \mathrm{C}$ and kept for 2 minutes, heated to $160^{\circ} \mathrm{C}$ for $\mathrm{HCs}$ and methyl ketones, or $220^{\circ} \mathrm{C}$ for fatty acid methyl esters (FAMEs) at a rate of $30^{\circ} \mathrm{C} / \mathrm{min}$, then increased at $3^{\circ} \mathrm{C} / \mathrm{min}$ up to $250^{\circ} \mathrm{C}$, followed by $10^{\circ} \mathrm{C} / \mathrm{min}$ up to $320^{\circ} \mathrm{C}$ and held for 5 minutes. Electron ionization mode $(70 \mathrm{eV})$ was used, and the MS scan range was 45 to $650 \mathrm{~m} / \mathrm{z}$ at a rate of 5 scans/s. Identification of HC compounds was performed according to Jurenka and colleagues [54], and peak area determination was performed with an Xcalibur 2.2 workstation.

\section{RNA isolation and real-time quantitative PCR (RT-qPCR)}

Total RNA was isolated with RNAiso Plus Reagents (Takara, Dalian, Liaoning, China) according to the manufacturer's instructions. cDNA was reverse-transcribed from $800 \mathrm{ng}$ of total RNA using the PrimeScrip RT reagent Kit with gDNA Eraser (Takara). Gene-specific primers with appropriate amplification efficiency ( 0.95 to 1.05$)$ were screened by a cDNA dilution series (S4 Table). Quantification of gene expression level was performed with TB Green Premix Ex Taq Tli RNase H Plus (Takara) on a LightCycler 480 system (Roche, Basle, Switzerland). Target genes expression was normalized by the commonly used housekeeping gene actin5c (GenBank: AJ862721.1) and calculated using the $2^{-\Delta \Delta C t}$ method. Each treatment contained 4 biological replicates and technical triplicates.

\section{Identification of BgElo gene family members}

Both BLASTN and BLASTP were used to search BgElo genes in B. germanica genome data [57] and our own full-length transcriptome data (NCBI accessions: SRR9143014 and SRR9143013) using the homologous genes of Elongase from D. melanogaster as query sequences. Candidate $B g E l o$ genes were amplified with the PrimeSTAR GXL DNA Polymerase reagent (Takara) (primers are listed in $\$ 4$ Table), and the amplified fragments were inserted into the PMD 19-T Vector (Takara) and re-sequenced. Candidate BgElo genes were then translated and submitted to SMART online tools (http://smart.embl-heidelberg.de) to analyze the conserved structures; only genes with the typical ELO domain were confirmed as BgElo genes. The putative BgElo mRNA sequences were mapped to the genomic data (GenBank: PYGN00000000.1) using a local BLASTN tool, and the intron-exon structure was analyzed based on the GT-AG rule. The conservative motifs in BgElos were analyzed by sequence alignment with DNAMAN 9.0 software.

\section{Expression profile analysis}

In order to screen the potential BgElo genes involved in HC biosynthesis, the expression levels of different BgElo genes were quantified in the fat body and abdominal integument, where HCs or their precursors were generated. In addition, other tissues including the head, thorax, gut, legs, ovaries, Malpighian tubules, ejaculatory duct (from 2-day-old males), and colleterial glands were dissected from 2-day-old females and were used to analyze the expression profiles of BgElo12 and BgElo24 among different tissues. In order to study the time course of BgElo12, $B g E l o 24, B g D T r a$, and $B g D s x$ transcript levels during sexual maturation, a representative nymphal stage (4-day-old sixth-instar nymph, N6D4) and AD0 to AD6 (0-day-old to 6-dayold adult) females and males were collected. Total RNA was extracted from various tissues or intact cockroaches, and the expression profiles of different genes were studied via RT-qPCR.

\section{Transcript knockdown via RNAi}

Gene-specific target sequences as well as a heterologous fragment from Mus musculus (Muslta) used for dsRNA synthesis were amplified and cloned into pMD 19-T Vector (Takara). 
Templates used for single-stranded RNA were amplified with primers that incorporated with the T7 promoter sequence ( $\$ 4$ Table). Different kinds of dsRNA were subsequently generated with the T7 RiboMAX Express RNAi System (Promega, Madison, Wisconsin, USA). Delivery of dsRNA was performed with a Nanoject II micro-injector (Drummond Scientific, Broomall, PA, USA) for fourth-instar cockroaches and microliter syringes for fifth-, sixth-instar, and adult cockroaches. For RNAi screen of BgElo genes in HC biogenesis, a double-injection strategy was employed: The first injection was performed at the early fifth-instar (1- or 2-day-old fifth-instar, N5D1-N5D2) with a dosage of $3 \mu \mathrm{g}$ in $2 \mu \mathrm{L}$; the second injection was performed 1 week later with a dosage of $4 \mu \mathrm{g}$ in $2 \mu \mathrm{L}$. For confirming the function of BgElo12 in contact sex pheromone biosynthesis, a third injection with $4 \mu \mathrm{g}$ of dsRNA in $2 \mu \mathrm{L}$ was carried out on (AD1, and methyl ketones were extracted at AD6. Knockdown of sex determination genes was accomplished with 3 dsRNA injections, the first at early fourth instar (N4D1 to N4D2), the second at early fifth instar (N5D1-N5D2), and the last at early sixth instar (N6D1 to N6D2). The fourth-instar cockroaches were injected with approximately $0.5 \mu \mathrm{g}$ of dsRNA in approximately $0.2 \mu \mathrm{L}$; the fifth- and sixth-instar cockroaches were injected with $1 \mu \mathrm{g}$ of dsRNA in $2 \mu \mathrm{L}$. In order to verify the function of BgElo12 and BgElo24 in HC biosynthesis and sex determination genes in modulating BgElo12 mRNA levels or HC profiles, 2 nonoverlapping genespecific targets were designed and used in this study; as there are some isoforms of $B g D s x$ and $B g T r a$, the RNAi targets were designed within the common sequence region. After dsRNA injection, intact $\mathrm{AD} 2$ cockroaches were used for RNAi efficiency analysis.

\section{Heterologous expression and fatty acid analysis}

Heterologous expression was performed as described [84]. Complete coding sequences (CDSs) of BgElo12, BgElo24, or control (GFP) were amplified with PrimeSTAR HS DNA Polymerase (Takara) using gene-specific primers ( $S 4$ Table) that contain the restriction enzyme sites (KpnI and BamHI for BgElo12 and BgElo24; BmHI and EcoRI for GFP) and the yeast consensus sequence (TACACA) following the restriction enzyme sites (only for forward primers). The amplified BgElo12, BgElo24, or GFP CDS fragments were ligated into the linearized pYES2 shuttle plasmid (Thermo Fisher Scientific) and verified by sequencing. The recombinant plasmids were transformed into INVSc1 S. cerevisiae (Thermo Fisher Scientific) using the PEG-LiAc method and streaked onto S. cerevisiae minimal medium minus uracil (SC-uracil) plates to select transformants; single colonies were inoculated in SC-uracil medium with $2 \%$ glucose. After culturing at $30^{\circ} \mathrm{C}$ for 24 hours, the yeast was collected and diluted to an OD600 of 0.4 with SC-uracil medium containing $1 \%$ raffinose and $2 \%$ galactose and further cultured at $30^{\circ} \mathrm{C}$ until they reached an OD600 of 0.8 . At this point, transcription of exogenous genes was examined by real-time PCR (RT-PCR). Substrates of C20 (0.5 mM), C22 (1 mM), C24 (1 $\mathrm{mM}), \mathrm{C} 26(1 \mathrm{mM}), \mathrm{C} 28(1 \mathrm{mM}), 2$-methylhexadecanoic acid $(0.5 \mathrm{mM})$, and 14-methylhexadecanoic acid $(0.5 \mathrm{mM})$ were separately added into the cultures with an extra $1 \%$ of tergitol type Nonidet P-40. All the substrates were purchased from Sigma-Aldrich (Louis, Missouri, USA) or TCI (Shanghai, China).

After 48 hours, yeast cells were harvested by centrifugation at $500 \mathrm{~g}$ for 5 minutes and washed thrice with Hanks' balanced salt solution for fatty acid derivatization and analysis. Pellets were dried under a steam of nitrogen, and $2 \mathrm{~mL}$ of $1 \%(\mathrm{v} / \mathrm{v}) \mathrm{H}_{2} \mathrm{SO}_{4}$ in methanol was added, and the mixture was vortexed and incubated at $80^{\circ} \mathrm{C}$ in a $\mathrm{N}_{2}$ atmosphere for 2 hours [85]. After that, $1 \mathrm{ml}$ of saturated sodium chloride solution was added into the mixture, and FAMEs were extracted with $1 \mathrm{~mL}$ of hexane 3 times. The FAME extracts were concentrated and subjected to GC-MS analysis as described above. Different FAMEs were identified by comparison to FAME standards (purchased from Sigma-Aldrich) and their mass spectra. 


\section{Desiccation bioassay}

The capacity of BgElo12 and BgElo24 to contribute to water retention was assessed by a desiccation bioassay. Drying bottles were prepared by putting approximately $120 \mathrm{~g}$ of packed fresh silica gel into an approximately $900-\mathrm{mL}$ sealed plastic bottle. The RH inside the bottle dropped to $5 \%$ within 2 hours, which was monitored by HOBO Pro v2 (Onset, Bourne, Massachusetts, USA). AD2 females were injected with dsBgElo12, dsBgELo24, and dsMuslta and separately caged in the desiccation bottles at $30^{\circ} \mathrm{C}$, supplied with approximately $1 \mathrm{~g}$ of dry food, but no water. Survival was recorded every 8 hours until all the cockroaches died. About 100 cockroaches were used for each treatment.

\section{Courtship behavioral study}

Courtship behavior was tested as described [86,87]. We firstly used antennae from AD5 females; each antenna was attached to the tip of a glass Pasteur pipette with paraffin, and the antenna was used immediately to test the responses of AD13 to AD15 males that were separated from females since eclosion. The test antenna was used to touch the antennae of the male, and a positive response was recorded if the male cockroach turned its body and raised the wings to approximately 90 degrees within 30 seconds. A negative response was recorded if the test antenna failed to elicit a response in a male cockroach and this male then responded to a positive control antenna from a normal AD6 female. The WR latency was recorded according to Wada-Katsumata and Schal [86]; the latency of the WR display was timed from contact of the antennae to the initiation of the male WR display. All female antennae and male cockroaches were used only once; all tests were performed in the scotophase, and we avoided the first and last 2 hours of the scotophase. Bioassays were conducted under a dim red light to simulate a dark environment. However, as AD5 females accumulated a large amount of contact sex pheromone, whereas males can be fully activated by about $10 \mathrm{ng}$ of 3,11-DimeC29-2-one applied onto an antenna [88], we repeated this experiment using AD3 females, which have less contact sex pheromone on the cuticle.

\section{Dual-luciferase reporter gene assay}

The $5^{\prime}$ end of BgElo12 was obtained by $5^{\prime}$ Rapid Amplification of cDNA Ends (RACE). The $5^{\prime}$ RACE cDNA library was prepared using Clontech SMARTer RACE 5'/3' Kit (Takara) according to the user manual with the gene-specific primer ( $\$ 4$ Table) and kit-provided Universal long primer. The amplified fragments were cloned into pRACE vector and sequenced. About a 2.7-kb sequence upstream of BgElo12 was amplified and cloned into pGL3-basic vector, and the CDS sequences of $B g D s x^{M}$ and GFP (control) were separately cloned into the expression vector pCDNA3.1. The HEK293T cells were cultured in a 24-well plate with $500 \mu \mathrm{L}$ of Dulbecco's Modified Eagle Medium (DMEM) (Thermo Fisher Scientific) for 24 hours before transfection, and the restructured pGL3-basic vector ( $200 \mathrm{ng} /$ well) was co-transfected with the expression vectors (200 ng/well) to HEK293T cells using Lipofectamine 3000 (Invitrogen, Carlsbad, California, USA). The pRL-TK that encoded a Renilla luciferase was also co-transfected as an internal control. The transfected cells were cultured at $37^{\circ} \mathrm{C}$ for 36 hours and subjected to luciferase activity analysis using the Dual-Glo Luciferase Assay System (Promega).

\section{Statistics}

Data were statistically analyzed using SPSS 23 and presented as mean \pm SEM or mean \pm SD . Two-tailed Student $t$ test was used for 2-group comparison; significant differences between multigroups were analyzed by 1-way ANOVA followed by the least significant difference 
(LSD) test (equal variances assumed) or Welch ANOVA followed by Games-Howell multiple comparisons test (equal variances not assumed) at $P<0.05$ level. PCA was used to distinguish the $\mathrm{CHC}$ profiles of AD1 and AD6 cockroaches.

\section{Supporting information}

S1 Fig. CHC profiles of 4-day-old sixth-instar nymphs of Blattella germanica. Peak 24 represents the female-enriched 3,7-; 3,9-; and 3,11-DimeC29, and Peak 17 is the male-enriched 9-; 11-; 13-; and 15-MeC29. The data underlying this figure are included in S2 Data. CHC, cuticular hydrocarbon.

S2 Fig. RNAi efficiency of different BgElo genes in Blattella germanica. Data are shown as mean \pm SEM, calculated from 3 to 4 replicates (2-3 cockroaches/replicate); ${ }^{* *} P<0.01,2$-tailed Student $t$ test. The data underlying this figure are included in S2 Data. RNAi, RNA interference.

(TIF)

S3 Fig. Effects of BgElo-RNAi on CHCs of Blattella germanica with chain length longer than 30. Different letters indicate significant differences between groups using Welch ANOVA (Games-Howell multiple comparisons test, $P<0.05$ ). The data underlying this figure are included in S2 Data. CHC, cuticular hydrocarbon; RNAi, RNA interference. (TIF)

S4 Fig. Effects of BgElo12-RNAi and BgElo24-RNAi on CHC profiles of B. germanica. (A, B) Relative amount of individual CHCs in males after knockdown of BgElo12 and BgElo24. Data are shown as mean $\pm \mathrm{SEM} ;{ }^{*} P<0.05,{ }^{* *} P<0.01 ; 2$-tailed Student $t$ test, $n=9$ or 10 . (C) PCA of CHC profiles after repression of BgElo12 and BgElo24; each dot represents a datum calculated from one cockroach. (D) The corresponding loading diagram; the numeric sequence labels correspond to numbers and CHC components in S2 Data. The data underlying this figure are included in S2 Data. CHC, cuticular hydrocarbon; PCA, principal component analysis; RNAi, RNA interference.

(TIF)

S5 Fig. Verifying the roles of BgElo12 and BgElo24 in $\mathrm{HC}$ biosynthesis by the second RNAi targets. (A) Analysis of CHCs after RNAi of BgElo12 using the second target (dsBgElo12B) (B) or after RNAi of BgElo24 using the second target (dsBgElo24B). (C, D) The effects of BgElo12RNAi and BgElo24-RNAi on internal HCs. Data are shown as mean \pm SEM; ${ }^{*} P<0.05$, ${ }^{* *} P<0.01 ; 2$-tailed Student $t$ test, $n=9-12$. The data underlying this figure are included in $\mathrm{S} 2$ Data. CHC, cuticular hydrocarbon; HC, hydrocarbon; RNAi, RNA interference.

S6 Fig. Tissue-specific expression of BgElo12 and BgElo24 in Blattella germanica. Data are shown as mean \pm SEM; and each sample was collected from 4 (AC, Th, and $\mathrm{Gu}$ ), 8 (FB, He, Ov, CG, and ED), and 12 (MT) cockroaches. Different letters indicate significant differences between groups using Welch ANOVA (Games-Howell multiple comparisons test, $P<0.05$ ), $n=4$. The data underlying this figure are included in S2 Data. AC, abdominal cuticle; Cg, colleterial gland; ED, ejaculatory duct; FB, fat body; Gu, gut; He, head; MT, Malpighian tubule; Ov, ovaries; Th, thorax.

(TIF) 
S7 Fig. Heterologous expression of BgElo12 and BgElo24 in yeast. RNAi of BgElo24 up-regulated the expression of BgElo12 (A), while RNAi of BgElo12 did not affect BgElo24 transcript level (B). Data are shown as mean \pm SEM; $P$ values were calculated from 4 samples; each sample contained 2 cockroaches; 2 -tailed Student $t$ test. (C) Detection of GFP protein in the yeast with pYES2-GFP using a FV3000 confocal fluorescence microscope (Olympus). (D, E) RT-PCR analysis of the BgElo12 and BgElo24 mRNA after the induction with galactose. (F, F', F") Gas chromatograms of FAMEs after adding 2-MeC16:0 into the medium. (G, G', G”) Gas chromatogram of FAMEs after adding 14-MeC16:0 into the medium. The compositions with retention times between 12 and 28 minutes were magnified about 50 times. (H, I) Mass spectra of methyl octacosanoate and methyl triacontanate, both of which showed a strong characteristic ion fragment $(\mathrm{m} / \mathrm{z}=74)$ and $\mathrm{M}$ peak. The data underlying S7A and S7B Fig are included in S2 Data. FAME, fatty acid methyl ester; RNAi, RNA interference; RT-PCR, real-time PCR. (TIF)

S8 Fig. The sex-specific developmental functions of BgTra and BgDsx in Blattella germanica. RNAi of BgTra in females generated a male-like body size and cuticle color, male-like tergal gland structure, and a protruding tissue at the end of the abdomen (left center); RNAi of $B g D s x$ in males generated a female-like body color and a protruding tissue at the end of the abdomen, and the tergal gland partly disappeared (right bottom). Other treatments did not generate obvious developmental effects. RNAi, RNA interference. (TIF)

S9 Fig. Transcriptional activity of $B g D s x^{M}$ on the upstream regulatory sequence of BgElo12. Data are shown as mean \pm SEM; $P$ values were calculated from 12 replicates; 2 -tailed Student $t$ test. The data underlying this figure are included in S2 Data. (TIF)

S10 Fig. Effects of $B g D s x$-RNAi in males and BgTra-RNAi in females on BgElo24 expression. Data are shown as mean \pm SEM; $P$ values were calculated from 4 replicates $(2$ cockroaches/replicate); 2 -tailed Student $t$ test. The data underlying this figure are included in S2 Data. RNAi, RNA interference.

S11 Fig. Regulation of cuticular hrdrocarbon profiles by sex differentiation genes. Data are shown as mean $\pm \mathrm{SEM},{ }^{*} P<0.05,{ }^{* *} P<0.01$, 2-tailed Student $t$ test, $n=14$ (dsMuslta-female), 12 (dsBgTra-female), 14 (dsBgTra+dsBgDsx-female), 12 (dsMuslta-male), and 16 (dsBgDsxmale). The data underlying this figure are included in S2 Data. (TIF)

S12 Fig. Regulation of internal hrdrocarbon profiles by sex differentiation genes. (A, B) Effects of $B g T r a-R N A i$ in females and $B g D s x$-RNAi in males on sex-specific internal HC profiles. (C, D) Proportion changes of representative internal HCs after RNAi of BgTra in females and $B g D s x$ in males. (E, F) Effects of BgTra-RNAi in females and $B g D s x$-RNAi in males on total amounts of internal HCs. Data are shown as mean \pm SEM, ${ }^{*} P<0.05,{ }^{* *} P<0.01,2$-tailed Student $t$ test, $n=10$ or 11. The data underlying this figure are included in S2 Data. HC, hydrocarbon; RNAi, RNA interference.

S1 Table. Quantification of difference $\mathrm{CHCs}$ during sexual dimorphic $\mathrm{CHCs}$ generation. CHC, cuticular hydrocarbon.

(DOCX) 
S2 Table. Quantification of individual CHC after RNAi of other BgElo genes. CHC, cuticular hydrocarbon; RNAi, RNA interference.

(DOCX)

S3 Table. Calculation the proportions of different FAMEs in yeast expression. FAME, fatty acid methyl ester.

(DOCX)

S4 Table. Primer sequences used in this study.

(DOCX)

S1 Appendix. Sequence alignment and protein structure analysis of BgElos. (DOCX)

S1 Data. The data underlying main figures (from Figs $1 \mathrm{~B}-1 \mathrm{E}, 2 \mathrm{~A}-2 \mathrm{~F}, 3 \mathrm{~B}-3 \mathrm{H}, 5 \mathrm{~A}, 5 \mathrm{C}-5 \mathrm{~F}$, $6 \mathrm{~A}-6 \mathrm{D}$, and $6 \mathrm{~F}-6 \mathrm{G})$.

(XLSX)

S2 Data. The data underlying Supporting information figures (from S2, S3, S4A, S4B, S5A-S5D, S6A, S6B, S7A, S7B, S9, S10, S11A, S11B, S12A, S12B, S12C, S12D, S12E, and S12F Figs).

(XLSX)

\section{Acknowledgments}

We thank Shang-Wang Hong from Northwest A \& F University for his help in sequence analysis and Hao-Su Cong and Professor Henry Chung from Michigan State University for their valuable suggestions.

\section{Author Contributions}

Conceptualization: Xiao-Jin Pei, Yong-Liang Fan.

Funding acquisition: Yong-Liang Fan, Coby Schal, Tong-Xian Liu.

Investigation: Xiao-Jin Pei, Yu Bai, Tian-Tian Bai, Zhan-Feng Zhang, Nan Chen.

Methodology: Xiao-Jin Pei, Yu Bai, Zhan-Feng Zhang, Nan Chen.

Project administration: Yong-Liang Fan, Coby Schal, Tong-Xian Liu.

Resources: Yong-Liang Fan, Coby Schal, Sheng Li, Tong-Xian Liu.

Supervision: Yong-Liang Fan, Tong-Xian Liu.

Writing - original draft: Xiao-Jin Pei.

Writing - review \& editing: Xiao-Jin Pei, Yong-Liang Fan, Yu Bai, Tian-Tian Bai, Coby Schal, Zhan-Feng Zhang, Nan Chen, Sheng Li, Tong-Xian Liu.

\section{References}

1. Bear A, Monteiro A. Both cell-autonomous mechanisms and hormones contribute to sexual development in vertebrates and insects. Bioessays. 2013; 35:725-32. https://doi.org/10.1002/bies.201300009 PMID: 23804281

2. Darwin C. The descent of man, and selection in relation to sex. London, UK: J. Murray. 1871.

3. Andersson M. Sexual selection. Princeton, NJ: Princeton University Press; 1994. 
4. Connallon T, Knowles LL. Intergenomic conflict revealed by patterns of sex-biased gene expression. Trends Genet. 2005; 21:495-9. https://doi.org/10.1016/j.tig.2005.07.006 PMID: 16039005

5. Innocenti $\mathrm{P}$, Morrow EH. The sexually antagonistic genes of Drosophila melanogaster. PLoS Biol. 2010; 8:e1000335. https://doi.org/10.1371/journal.pbio.1000335 PMID: 20305719

6. Mank JE. The transcriptional architecture of phenotypic dimorphism. Nature Ecol Evol. 2017; 1(6). https://doi.org/10.1038/s41559-016-0006 PMID: 28812569

7. Rogers TF, Palmer DH, Wright AE. Sex-specific selection drives the evolution of alternative splicing in birds. Mol Biol Evol. 2021; 38:519-30. https://doi.org/10.1093/molbev/msaa242 PMID: 32977339

8. Kopp A. Dmrt genes in the development and evolution of sexual dimorphism. Trends Genet. 2012; 28:175-84. https://doi.org/10.1016/j.tig.2012.02.002 PMID: 22425532

9. Williams TM, Selegue JE, Werner T, Gompel N, Kopp A, Carroll SB. The regulation and evolution of a genetic switch controlling sexually dimorphic traits in Drosophila. Cell. 2008; 134:610-23. https://doi. org/10.1016/j.cell.2008.06.052 PMID: 18724934

10. Tanaka K, Barmina O, Sanders LE, Arbeitman MN, Kopp A. Evolution of sex-specific traits through changes in HOX-dependent doublesex expression. PLoS Biol. 2011; 9:e1001131. https://doi.org/10 1371/journal.pbio.1001131 PMID: 21886483

11. Clough E, Jimenez E, Kim YA, Whitworth C, Neville MC, Hempel LU, et al. Sex- and tissue-specific functions of Drosophila doublesex transcription factor target genes. Dev Cell. 2014; 31:761-73. https:// doi.org/10.1016/j.devcel.2014.11.021 PMID: 25535918

12. Prakash A, Monteiro A. Molecular mechanisms of secondary sexual trait development in insects. Curr Opin Insect Sci. 2016; 17:40-8. https://doi.org/10.1016/j.cois.2016.06.003 PMID: 27720072

13. Hasselmann M, Gempe T, Schiøtt M, Nunes-Silva CG, Otte M, Beye M. Evidence for the evolutionary nascence of a novel sex determination pathway in honeybees. Nature. 2008; 454:519-22. https://doi. org/10.1038/nature07052 PMID: 18594516

14. Zhang Z, Klein J, Nei M. Evolution of the sex-lethal gene in insects and origin of the sex-determination system in Drosophila. J Mol Evol. 2014; 78:50-65. https://doi.org/10.1007/s00239-013-9599-3 PMID: 24271947

15. Kiuchi $\mathrm{T}$, Koga H, Kawamoto M, Shoji $\mathrm{K}$, Sakai $\mathrm{H}$, Arai $\mathrm{Y}$, et al. A single female-specific piRNA is the primary determiner of sex in the silkworm. Nature. 2014; 509:633-6. https://doi.org/10.1038/nature13315 PMID: 24828047

16. Herpin A, Schartl M. Plasticity of gene-regulatory networks controlling sex determination: of masters, slaves, usual suspects, newcomers, and usurpators. EMBO Rep. 2015; 16:1260-1274. https://doi.org/ 10.15252/embr.201540667 PMID: 26358957

17. Hall $A B$, Basu S, Jiang X, Qi Y, Timoshevskiy VA, Biedler JK, et al. A male-determining factor in the mosquito Aedes aegypti. Science. 2015; 348:1268-70. https://doi.org/10.1126/science.aaa2850 PMID: 25999371

18. Gibbs AG. Water-proofing properties of cuticular lipids. Am Zool. 1998; 38:471-82. https://doi.org/10. 1093/icb/38.3.471

19. Blomquist GJ, Ginzel MD. Chemical ecology, biochemistry, and molecular biology of insect hydrocarbons. Annu Rev Entomol. 2021; 66:45-60. https://doi.org/10.1146/annurev-ento-031620-071754 PMID: 33417824

20. Howard RW, Blomquist GJ. Ecological, behavioral, and biochemical aspects of insect hydrocarbons. Annu Rev Entomol. 2005; 50:371-93. https://doi.org/10.1146/annurev.ento.50.071803.130359 PMID: 15355247

21. Ingleby FC, Innocenti $P$, Rundle HD, Morrow EH. Between-sex genetic covariance constrains the evolution of sexual dimorphism in Drosophila melanogaster. J Evol Biol. 2014; 27:1721-32. https://doi.org/ 10.1111/jeb.12429 PMID: 24893565

22. Zhang B, Xue HJ, Song KQ, Liu J, Li WZ, Nie RE, et al. Male mate recognition via cuticular hydrocarbons facilitates sexual isolation between sympatric leaf beetle sister species. J Insect Physiol. 2014; 70:15-21. https://doi.org/10.1016/j.jinsphys.2014.08.006 PMID: 25172230

23. Berson JD, Garcia-Gonzalez F, Simmons LW. Experimental evidence for the role of sexual selection in the evolution of cuticular hydrocarbons in the dung beetle, Onthophagus taurus. J Evol Biol. 2019; 32:1186-93. https://doi.org/10.1111/jeb.13519 PMID: 31420906

24. Juárez MP, Ayala S, Brenner RR. Methyl-branched fatty acid biosynthesis in Triatoma infestans. Insect Biochem Mol Biol. 1996; 26:599-605. https://doi.org/10.1016/S0965-1748(96)00021-5

25. Juárez MP, Chase J. Blomquist GJ. A microsomal fatty acid synthetase from the integument of Blattella germanica synthesizes methyl-branched fatty acids, precursors to hydrocarbon and contact sex pheromone. Arch Insect Biochem Physiol. 1992; 293:333-41. https://doi.org/101016/0003-9861(92)90403-j 
26. Blomquist GJ, Tillman JA, Reed JR, Gu P, Vanderwel D, Choi S, et al. Regulation of enzymatic activity involved in sex pheromone production in the housefly, Musca domestica. Insect Biochem Mol Biol. 1995; 25:751-7. https://doi.org/10.1016/0965-1748(95)00015-n PMID: 7627207

27. Parvy JP, Napal L, Rubin T, Poidevin M, Perrin L, Wicker-Thomas C, et al. Drosophila melanogaster Acetyl-CoA-carboxylase sustains a fatty acid-dependent remote signal to waterproof the respiratory system. PLoS Genet. 2012; 8:e1002925. https://doi.org/10.1371/journal.pgen.1002925 PMID: 22956916

28. Chung H, Loehlin DW, Dufour HD, Vaccarro K, Millar JG. Carroll SB. A single gene affects both ecological divergence and mate choice in Drosophila. Science. 2014; 343:1148-51. https://doi.org/10.1126/ science.1249998 PMID: 24526311

29. Wicker-Thomas C, Garrido D, Bontonou G, Napal L, Mazuras N, Denis B, et al. Flexible origin of hydrocarbon/pheromone precursors in Drosophila melanogaster. J Lipid Res. 2015; 56:2094-101. https:// doi.org/10.1194/jlr.M060368 PMID: 26353752

30. Ginzel MD, Blomquist GJ. Insect hydrocarbons: biochemistry and chemical ecology. In: Cohen E, Moussian B, editors. Extracellular Composite Matrices in Arthropods. Springer; 2016. pp. 221-252.

31. Chertemps T, Duportets L, Labeur C, Ueyama M, Wicker-Thomas C. A female specific desaturase gene responsible for diene hydrocarbon biosynthesis and courtship behaviour in Drosophila melanogaster. Insect Mol Biol. 2006; 15:465-73. https://doi.org/10.1111/j.1365-2583.2006.00658.x PMID: 16907833

32. Legendre A, Miao XX, Da Lage JL, Wicker-Thomas C. Evolution of a desaturase involved in female pheromonal cuticular hydrocarbon biosynthesis and courtship behavior in Drosophila. Insect Biochem Mol Biol. 2008; 38:244-55. https://doi.org/10.1016/j.ibmb.2007.11.005 PMID: 18207084

33. Chertemps T, Duportets L, Labeur C, Ueda R, Takahashi K, Saigo K, et al. A female-biased expressed elongase involved in long-chain hydrocarbon biosynthesis and courtship behavior in Drosophila melanogaster. Proc Natl Acad Sci U S A. 2007; 104:4273-4278. https://doi.org/10.1073/pnas.0608142104 PMID: 17360514

34. Qiu Y, Tittiger C, Wicker-thomas C, Goff LG, Young S, Wajnberg E, et al. An insect-specific p450 oxidative decarbonylase for cuticular hydrocarbon biosynthesis. Proc Natl Acad Sci U S A. 2012; 109:1485814863. https://doi.org/10.1073/pnas.1208650109 PMID: 22927409

35. MacLean M, Nadeau J, Gurnea T, Tittiger C, Blomquist GJ. Mountain pine beetle (Dendroctonus ponderosae) CYP4Gs convert long and short chain alcohols and aldehydes to hydrocarbons. Insect Biochem Mol Biol. 2018; 102:11-20. https://doi.org/10.1016/j.ibmb.2018.09.005 PMID: 30243802

36. Li DT, Chen X, Wang XQ, Zhang CX. FAR gene enables the brown planthopper to walk and jump on water in paddy field. Sci China Life Sci. 2019; 62:1521-31. https://doi.org/10.1007/s11427-018-9462-4 PMID: 30810963

37. Blomquist GJ, Bagnères AG. Insect hydrocarbons: biology, biochemistry, and chemical ecology. 1st ed. New York: Cambridge University Press; 2010.

38. Chung $\mathrm{H}$, Carroll SB. Wax, sex and the origin of species: Dual roles of insect cuticular hydrocarbons in adaptation and mating. Bioessays. 2015; 37:822-30. https://doi.org/10.1002/bies.201500014 PMID: 25988392

39. Holze H, Schrader L, Buellesbach J. Advances in deciphering the genetic basis of insect cuticular hydrocarbon biosynthesis and variation. Heredity. 2021; 126:219-34. https://doi.org/10.1038/s41437020-00380-y PMID: 33139902

40. Ferveur JF, Sureau G. Simultaneous influence on male courtship of stimulatory and inhibitory pheromones produced by live sex-mosaic Drosophila melanogaster. Proc R Soc B. 1996; 263:967-73. https://doi.org/10.1098/rspb.1996.0143 PMID: 8805834

41. Ferveur JF, Savarit F, O'Kane CJ, Sureau G, Greenspan RJ, et al. Genetic feminization of pheromones and its behavioral consequences in Drosophila males. Science. 1997; 276:1555-8. https://doi.org/10. 1126/science.276.5318.1555 PMID: 9171057

42. Shirangi TR, Dufour HD, Williams TM, Carroll SB. Rapid evolution of sex pheromone-producing enzyme expression in Drosophila. PLoS Biol. 2009; 7:e1000168. https://doi.org/10.1371/journal.pbio.1000168 PMID: 19652700

43. Schal C. Cockroaches. In: Hedges S, Moreland D, editors, Handbook of Pest Control. Pennsylvania State University: GIEMedia; 2011. pp. 150-291.

44. Chase J, Jurenka RA, Schal C, Halarnkar PP, Blomquist GJ. Biosynthesis of methyl branched hydrocarbons of the German cockroach Blattella germanica (L.) (Orthoptera, Blattellidae. Insect Biochem. 1990; 20:149-56. https://doi.org/10.1016/0020-1790(90)90007-H 
45. Schal C, Gu X, Burns EL, Blomquist GJ. Patterns of biosynthesis and accumulation of hydrocarbons and contact sex pheromone in the female German cockroach, Blattella germanica. Arch Insect Biochem Physiol. 1994; 25:375-91. https://doi.org/10.1002/arch.940250411 PMID: 8204906

46. Juárez MP. Fatty acyl-CoA elongation in Blatella germanica integumental microsomes. Arch Insect Biochem Physiol. 2004; 56:170-8. https://doi.org/10.1002/arch.20007 PMID: 15274178

47. Young HP, Larabee JK, Gibbs AG, Schal C. Relationship between tissue-specific hydrocarbon profiles and lipid melting temperatures in the cockroach Blattella germanica. J Chem Ecol. 2000; 26:12451263. https://doi.org/10.1023/A:1005440212538

48. Chase J, Touhara K, Prestwich GD, Schal C, Blomquist GJ. Biosynthesis and endocrine control of the production of the German cockroach sex pheromone 3,11-dimethylnonacosan-2-one. Proc Natl Acad Sci U S A. 1992; 89:6050-4. https://doi.org/10.1073/pnas.89.13.6050 PMID: 1631090

49. Nojima S, Nishida R, Kuwahara Y, Sakuma M. Nuptial feeding stimulants: a male courtship pheromone of the German cockroach, Blattella germanica (L.) (Dictyoptera: Blattellidae. Naturwissenschaften. 1999; 86:193-6. https://doi.org/10.1007/s001140050596

50. Eliyahu D, Nojima S, Mori K, Schal C. New contact sex pheromone components of the German cockroach, Blattella germanica, predicted from the proposed biosynthetic pathway. J Chem Ecol. 2008; 34:229-37. https://doi.org/10.1007/s10886-007-9409-8 PMID: 18231836

51. Eliyahu D, Nojima S, Mori K, Schal C. Jail baits: how and why nymphs mimic adult females of the German cockroach, Blattella germanica. Anim Behav. 2009; 78:1097-105. https://doi.org/10.1016/j. anbehav.2009.06.035

52. Wexler J, Delaney EK, Belles X, Schal C, Wada-Katsumata A, et al. Hemimetabolous insects elucidate the origin of sexual development via alternative splicing. Elife. 2019; 8:e47490. https://doi.org/10.7554/ eLife.47490 PMID: 31478483

53. Schal C, Chiang AS. Hormonal control of sexual receptivity in cockroaches. Experientia. 1995; 51:9948. https://doi.org/10.1007/BF01921755 PMID: 7556584

54. Jurenka RA, Schal C, Burns E, Chase J, Blomquist GJ. Structural correlation between cuticular hydrocarbons and female contact sex pheromone of German cockroach Blattella germanica (L.). J Chem Ecol. 1989; 15:939-49. https://doi.org/10.1007/BF01015189 PMID: 24271896

55. Pei XJ, Chen N, Bai Y, Qiao JW, Li S, et al. BgFas 1: A fatty acid synthase gene required for both hydrocarbon and cuticular fatty acid biosynthesis in the German cockroach, Blattella germanica (L.). Insect Biochem Mol Biol. 2019; 112:103203. https://doi.org/10.1016/j.ibmb.2019.103203 PMID: 31425851

56. Chen N, Pei XJ, Li S, Fan YL, Liu TX. Involvement of integument-rich CYP4G19 in hydrocarbon biosynthesis and cuticular penetration resistance in Blattella germanica (L.). Pest Manag Sci. 2020; 76:21526. https://doi.org/10.1002/ps.5499 PMID: 31149772

57. Harrison MC, Jongepier E, Robertson HM, Arning N, Bitard-Feildel T, Chao H, et al. Hemimetabolous genomes reveal molecular basis of termite eusociality. Nat Ecol Evol. 2018; 2:557-66. https://doi.org/ 10.1038/s41559-017-0459-1 PMID: 29403074

58. Moon YA, Shah NA, Mohapatra S, Warrington JA, Horton JD. Identification of a mammalian long chain fatty acyl elongase regulated by sterol regulatory element-binding proteins. J Biol Chem. 2001; 276:45358-66. https://doi.org/10.1074/jbc.M108413200 PMID: 11567032

59. Toke DA, Martin CE. Isolation and characterization of gene affecting fatty acid elongation in Saccharomyces cerevisiae. J Biol Chem. 1996; 271:18413-22. https://doi.org/10.1074/jbc.271.31.18413 PMID: 8702485

60. Oh CS, Toke DA, Mandala S, Martin CE. ELO2 and ELO3, Homologues of the Saccharomyces cerevisiae ELO1 gene, function in fatty acid elongation and are required for sphingolipid formation. J Boil Chem. 1997; 272:17376-84. https://doi.org/10.1074/jbc.272.28.17376 PMID: 9211877

61. Ohno Y, Suto S, Yamanaka M, Mizutani Y, Mitsutake S, Igarashi Y, et al. ELOVL1 production of C24 acyl-CoAs is linked to C24 sphingolipid synthesis. Proc Natl Acad Sci U S A. 2010; 107:18439-18444. https://doi.org/10.1073/pnas.1005572107 PMID: 20937905

62. Pfannenstiel RS, Booth W, Vargo EL, Schal C. Blattella asahinai (Dictyoptera: Blattellidae): A new predator of lepidopteran eggs in south Texas soybean. Ann Entomol Soc Am. 2008; 101:763-8.

63. Schal C, Sevala V, Capurro ML, Snyder TE, Blomquist GJ, Bagnères AG. Tissue distribution and lipophorin transport of hydrocarbons and sex pheromones in the house fly, Musca domestica. J Insect Sci. 2001; 1:12. PMID: 15455072

64. Schal C, Sevala V, Cardé RT. Novel and highly specific transport of a volatile sex pheromone by hemolymph lipophorin in moths. Naturwissenschaften. 1998; 85:339-42. https://doi.org/10.1007/ s001140050511 
65. Gu X, Quilici D, Juarez P, Blomquist GJ, Schal C. Biosynthesis of hydrocarbons and contact sex pheromone and their transport by lipophorin in females of the German cockroach (Blattella germanica). J Insect Physiol. 1995; 41:257-67. https://doi.org/10.1016/0022-1910(94)00100-U

66. Li DT, Chen X, Wang XQ, Moussian B, Zhang CX. The fatty acid elongase gene family in the brown planthopper, Nilaparvata lugens. Insect Biochem Mol Biol. 2019; 108:32-43. https://doi.org/10.1016/j. ibmb.2019.03.005 PMID: 30885803

67. Zhao X, Yang Y, Niu N, Zhao $Y$, Zhang J. The fatty acid elongase gene $L m E l o 7$ is required for hydrocarbon biosynthesis and cuticle permeability in the migratory locust, Locusta migratoria. J Insect Physiol. 2020; 123:104052. https://doi.org/10.1016/j.jinsphys.2020.104052 PMID: 32259526

68. Gempe T, Beye M. Function and evolution of sex determination mechanisms, genes and pathways in insects. Bioessays. 2011; 33:52-60. https://doi.org/10.1002/bies.201000043 PMID: 21110346

69. Verhulst EC, van de Zande L. Double nexus-Doublesex is the connecting element in sex determination. Brief Funct Genomics. 2015; 14:396-406. https://doi.org/10.1093/bfgp/elv005 PMID: 25797692

70. Wicker C, Jallon JM. Hormonal control of sex pheromone biosynthesis in Drosophila melanogaster. J Insect Physiol. 1995; 41:65-70. https://doi.org/10.1016/0022-1910(94)00074-Q

71. Wicker C, Jallon JM. Influence of ovary and ecdysteroids on pheromone biosynthesis in Drosophila melanogaster (Diptera: Drosophilidae). Eur J Entomol. 1995; 92:197202.

72. Marican C, Duportets L, Birman S, Jallon JM. Female-specific regulation of cuticular hydrocarbon biosynthesis by dopamine in Drosophila melanogaster. Insect Biochem Mol Biol. 2004; 34:823-30. https:// doi.org/10.1016/j.ibmb.2004.05.002 PMID: 15262286

73. Kuo TH, Fedina TY, Hansen I, Dreisewerd K, Dierick HA, Yew JY, et al. Insulin signaling mediates sexual attractiveness in Drosophila. PLoS Genet. 2012; 8:e1002684. https://doi.org/10.1371/journal.pgen. 1002684 PMID: 22570625

74. Bontonou G, Shaik HA, Denis B, Wicker-Thomas C. Acp70A regulates Drosophila pheromones through juvenile hormone induction. Insect Biochem Mol Biol. 2015; 56:36-49. https://doi.org/10.1016/j.ibmb. 2014.11.008 PMID: 25484200

75. Fedina TY, Arbuthnott D, Rundle HD, Promislow D, Pletcher SD. Tissue-specific insulin signaling mediates female sexual attractiveness. PLoS Genet. 2017; 13:e1006935. https://doi.org/10.1371/journal. pgen.1006935 PMID: 28817572

76. Baron A, Denis B, Wicker-Thomas C. Control of pheromone production by ovaries in Drosophila. J Insect Physiol. 2018; 109:138-43. https://doi.org/10.1016/j.jinsphys.2018.07.003 PMID: 30003888

77. Chiang YN, Tan KJ, Chung H, Lavrynenko O, Shevchenko A, Yew JY. Steroid hormone signaling is essential for pheromone production and oenocyte survival. PLoS Genet. 2016; 12:e1006126. https:// doi.org/10.1371/journal.pgen.1006126 PMID: 27333054

78. Bownes M, Smith T. Ecdysteroids in adult males and females of Drosophila melanogaster. J Insect Physiol. 1984; 30:823-30. https://doi.org/10.1016/0022-1910(84)90019-2

79. Romaá I, Pascual N, Bellés X. The ovary is a source of circulating ecdysteroids in Blattella germanica (Dictyoptera: Blattellidae). Eur J Entomol. 1995; 92:93-103.

80. Zinna RA, Gotoh H, Kojima T, Niimi T. Recent advances in understanding the mechanisms of sexually dimorphic plasticity: insights from beetle weapons and future directions. Curr Opin Insect Sci. 2018; 25:35-41. https://doi.org/10.1016/j.cois.2017.11.009 PMID: 29602360

81. Kunte K. Mimetic butterflies support Wallace's model of sexual dimorphism. Proc R Soc B. 2008; 275:1617-24. https://doi.org/10.1098/rspb.2008.0171 PMID: 18426753

82. Fan YL, Eliyahu D, Schal C. Cuticular hydrocarbons as maternal provisions in embryos and nymphs of the cockroach Blattella germanica. J Exp Biol. 2008; 211: 548-554. https://jeb.biologists.org/content/ 211/4/548. https://doi.org/10.1242/jeb.009233 PMID: 18245631

83. Fan YL, Chase J, Sevala VL, Schal C. Lipophorin-facilitated hydrocarbon uptake by oocytes in the German cockroach Blattella germanica (L.). J Exp Biol. 2002; 205:781-790. https://jeb.biologists.org/ content/205/6/781. PMID: 11914386

84. Hastings N, Agaba M, Tocher DR, Leaver MJ, Dick JR, Sargent JR, et al. A vertebrate fatty acid desaturase with Delta 5 and Delta 6 activities. Proc Natl Acad Sci U S A. 2001; 98:14304-14309. https://doi. org/10.1073/pnas.251516598 PMID: 11724940

85. Lepage G, Roy C. Improved recovery of fatty acid through direct transesterification without prior extraction or purification. J Lipid Res. 1984; 25:1391-6. PMID: 6530596

86. Wada-Katsumata A, Schal C. Antennal grooming facilitates courtship performance in a group-living insect, the German cockroach Blattella germanica. Sci Rep. 2019; 9:2942. https://doi.org/10.1038/ s41598-019-39868-x PMID: 30814635 
87. Eliyahu D, Nojima S, Capracotta SS, Comins DL, Schal C. Identification of cuticular lipids eliciting interspecific courtship in the German cockroach, Blattella germanica. Naturwissenschaften. 2008; 95:40312. https://doi.org/10.1007/s00114-007-0339-7 PMID: 18183359

88. Schal C, Burns EL, Jurenka RA, Blomquist GJ. A new component of the female sex pheromone of Blattella germanica (L.) (Dictyoptera: Blattellidae) and interaction with other pheromone components. J

Chem Ecol. 1990; 16:1997-2008. https://doi.org/10.1007/BF01020511 PMID: 24264001 